\title{
Hot gas and magnetic arms of NGC 6946: Indications for reconnection heating? ${ }^{\star}$
}

\author{
M. Weżgowiec ${ }^{1,2}$, M. Ehle ${ }^{3}$, and R. Beck ${ }^{4}$ \\ 1 Astronomisches Institut der Ruhr-Universität Bochum, Universitätsstrasse 150, 44780 Bochum, Germany \\ e-mail: markmet@oa.uj.edu.pl \\ 2 Obserwatorium Astronomiczne Uniwersytetu Jagiellońskiego, ul. Orla 171, 30-244 Kraków, Poland \\ 3 ESA-ESAC, XMM-Newton Science Operations Centre, PO Box 78, 28691 Villanueva de la Cañada, Madrid, Spain \\ 4 Max-Planck-Institut für Radioastronomie, Auf dem Hügel 69, 53121 Bonn, Germany
}

Received 25 June 2015 / Accepted 15 October 2015

\section{ABSTRACT}

\begin{abstract}
Context. The grand-design face-on spiral galaxy NGC 6946 is remarkable because of its high star formation activity, the massive northern spiral arm, and the magnetic arms, which are observed in polarized radio synchrotron emission and are located between the optical arms and possibly are magnetic reconnection regions.

Aims. We used electron densities and temperatures in star-forming (active) and less active regions and compared them to findings from the analysis of the radio data to study the energy budget of NGC 6946. The hot gas above the magnetic arms between the optical arms might suggest gas heating by reconnection. We also study the population of point sources in NGC 6946, including the origin of the puzzling ultra-luminous emission complex MF16.

Methods. X-ray observations of NGC 6946 performed with XMM-Newton were used to study the emission from X-ray point sources and diffuse hot gas, including the magnetic arms and the halo. Spectral fitting of the diffuse X-ray emission allowed us to derive temperatures of the hot gas. With assumptions about the emission volume, this allowed us to estimate gas densities, masses, and cooling times.

Results. To explain the X-ray emission from the spiral arms of NGC 6946 two-temperature plasma models are needed to account for the disk and halo emission. The interarm regions show only one thermal component. We observe that the temperature of the hot gas in and above the magnetic arm regions increases slightly when compared to the average temperatures in the areas in and above the spiral arms. For the southwestern part of the disk, which is depolarized in the radio range by Faraday rotation, we find more efficient mixing of disk and halo gas.

Conclusions. We propose magnetic reconnection in the magnetic arm regions of NGC 6946 as the possible cause of the additional heating of the gas and ordering of the magnetic fields. In the southwestern part of the galactic disk we observed indications of a possible faster outflow of the hot gas. A very hot gas within the MF 16 nebula possibly suggests shock heating by a supernova explosion.
\end{abstract}

Key words. galaxies: individual: NGC 6946 - galaxies: ISM - galaxies: spiral - galaxies: magnetic fields

\section{Introduction}

NGC 6946 (see Table 1) is a Scd spiral galaxy seen face-on, which is listed in Arp's atlas of peculiar galaxies because of its massive northern spiral arm. Its large optical diameter and low inclination make NGC 6946 one of the most prominent granddesign spiral galaxies in the sky. It is known to host a bright starburst nucleus (see e.g. Telesco \& Harper 1980). Detections of CO radio emission lines (Nieten et al. 1999; Walsh et al. 2002) gave further evidence for the high star-forming activity of NGC 6946 (especially in the northern spiral arm), proved that molecular clouds containing warm and dense gas are distributed throughout the inner disk, and showed that the total molecular gas mass is exceptionally high for a spiral galaxy. The distributions of the emission from NGC 6946 in various spectral ranges were analysed with wavelet functions (see Frick et al. 2000).

\footnotetext{
* Based on observations obtained with XMM-Newton, an ESA science mission with instruments and contributions directly funded by ESA Member States and NASA.
}

Table 1. Basic astronomical properties of NGC 6946.

\begin{tabular}{lc}
\hline \hline Morphological type & SABc \\
Inclination & $18^{\circ}$ \\
Diameter $\mathrm{D}_{25}$ & $11^{\prime} .5$ \\
$\mathrm{RA}_{2000}$ & $20^{\mathrm{h}} 34^{\mathrm{m}} 53^{\mathrm{s}}$ \\
Dec $_{2000}$ & $+60^{\circ} 09^{\prime} 13^{\prime \prime}$ \\
Distance $^{a}$ & $7 \mathrm{Mpc}$ \\
\hline
\end{tabular}

Notes. All data except for the distance are taken from the HYPERLEDA database - http://leda.univ-lyon1.fr - see Paturel (2003). (a) Taken from Israel (1980).

\subsection{Previous radio continuum findings}

NGC 6946 has been thoroughly studied in the radio regime in total and polarized emission (Beck 1991; Ehle \& Beck 1993). Radio polarization observations at $18 \mathrm{~cm}$ and $20 \mathrm{~cm}$ wavelengths revealed excess Faraday rotation and strong depolarization in the SW quadrant of NGC 6946 that is probably due to a large-scale magnetic field along the line of sight, oriented approximately 
perpendicular to the disk plane (Beck 1991). Analysis of polarization data at four frequencies also suggested strong vertical fields extending far above the disk (Beck 2007). Such field lines should enable an outflow of hot gas into a halo. As the SW quadrant of NGC 6946 is a region of relatively low star-formation activity, this phenomenon resembles a coronal hole on the Sun.

The average energy density of the warm ionized medium in the interstellar medium (ISM) of the inner disk of NGC 6946 was found to be lower by a factor of about 10 than the energy densities of the magnetic field and that of the cosmic rays, resulting in the conclusion that the magnetic field dominates thermal processes in the disk and halos of galaxies (Beck 2004). A significant fraction of the diffuse ISM must be unstable, giving rise to gas outflows.

Radio polarization data at $\lambda 6 \mathrm{~cm}$ led to the discovery of a new phenomenon: highly aligned magnetic fields that are concentrated in two main spiral features, located almost precisely between the optical spiral arms of NGC 6946 (Beck \& Hoernes 1996, their Fig. 1). No enhanced densities of molecular, neutral, or warm ionized gas have been detected at the positions of these magnetic arms. However, radio observations revealed significant Faraday rotation in these regions so that some ionized gas must be present. Frick et al. (2000) analysed the magnetic and optical spiral arms in NGC 6946 using 1D wavelet transformations and found that each magnetic arm is similar to the preceding optical arm and hence can be regarded as a phase-shifted image.

Rudimentary magnetic arms were also found in other spiral galaxies (Beck 2015), but NGC 6946 still is the most prominent example.

The magnetic arms contradict density-wave models, which predict enhanced ordered magnetic fields at the inner edges of the arms. Several mechanisms were proposed to explain the magnetic arms. For example, the continuous injection and amplification of turbulent fields by supernova shock fronts may suppress the mean-field dynamo in the material arms (Moss et al. 2013, 2015). Alternatively, the introduction of a relaxation time of the magnetic response in the dynamo equation may lead to a phase shift between the material and magnetic spiral arms (Chamandy et al. 2013a,b). The mean-field dynamo in the material arms might also be suppressed by outflows driven by star formation (Chamandy et al. 2015).

We propose here that the strongly polarized radio emission from the magnetic arms may also suggest the existence of reconnection regions where cosmic rays are accelerated. The gas heated by the same process should be detectable in the X-ray domain (Lesch \& Bender 1990; Hanasz \& Lesch 2003). By comparing the properties of the hot gas in the magnetic and spiral arms, we may be able to trace a possible additional heating of the gas that would be caused by the reconnection processes.

\subsection{Earlier $X$-ray observations}

Despite the rather high $N_{\mathrm{H}}$-value of about $2 \times 10^{21} \mathrm{~cm}^{-2}$ (see Table 2), NGC 6946 was detected by ROSAT's All Sky survey with a count rate of $0.1 \mathrm{cts} \mathrm{s}^{-1}$. Analysing the $36 \mathrm{ks}$ ROSAT PSPC pointed observation, Schlegel (1994a,b,c) apart from SN 1980K additionally reported emission from nine pointlike X-ray sources and diffuse emission from NGC 6946. The brightest source (identified at that time with a very luminous supernova remnant MF16) has a count rate of $0.07 \mathrm{cts} \mathrm{s}^{-1}$ corresponding to a flux of $8.3 \times 10^{-13} \mathrm{erg} \mathrm{cm}^{-2} \mathrm{~s}^{-1}$ in the energy range $0.5-2 \mathrm{keV}$, three sources are fainter by about a factor of 10 , and the rest (fainter by a factor of 40) are at the detection threshold.
Table 2. Characteristics of the XMM-Newton X-ray observations of NGC 6946.

\begin{tabular}{lc}
\hline \hline Obs ID & 0200670301 \\
& 0500730101 \\
& 0500730201 \\
& 0691570101 \\
column density $N_{\mathrm{H}}{ }^{a}$ & 1.84 \\
MOS filter & medium \\
MOS obs. mode & $\mathrm{FF}$ \\
pn filter & medium \\
pn obs. mode & $\mathrm{FF}$ \\
Total/clean pn time $[\mathrm{ks}]$ & $13.1 / 8.3$ \\
& $28.4 / 20.2$ \\
& $33.3 / 29.7$ \\
& $114.3 / 98.2$ \\
\hline
\end{tabular}

Notes. ${ }^{(a)}$ Column density in $\left[10^{21} \mathrm{~cm}^{-2}\right]$ weighted average value after LAB Survey of Galactic H I Kalberla et al. (2005).

Holt et al. (2003) studied discrete X-ray sources in NGC 6946 using a 60 ks Chandra ACIS observation and found the source population dominated by high-mass X-ray binaries. Their survey was complete down to approximately $10^{37} \mathrm{erg} \mathrm{s}^{-1}$. However, in contrast to previous results, the ultra-luminous MF16 complex was found to be deficient in line emission expected from an interaction with a dense surrounding medium. Its spectrum lacks pronounced spectral lines and can be fit with a variety of models that are all associated with unusually high luminosities, leaving the origin of the MF16 related X-ray emission unknown. Schlegel et al. (2003) used the same Chandra observations and the luminosity function derived by Holt et al. (2003) to distinguish point sources from the diffuse emission. These authors estimated that as much as $10 \%$ of the total soft $\mathrm{X}$-ray emission could be due to a hot diffuse component.

We checked the Chandra archive and found additional ACIS-S pointings: one on-axis pointing aiming at $\mathrm{SN} 2002 \mathrm{HH}$ (30 ks, IAU Circ. 8024 and Roberts \& Colbert 2003), and three (30 ks each) off-axis pointings centred on SN2004et. The combination of these Chandra observations was used by severeal authors (Soria \& Perna 2008; Fridriksson et al. 2008; Kajava \& Poutanen 2009; Liu 2011) to investigate individual sources (e.g. supernovae, ultra-luminous sources) in NGC 6946 and their spectral and temporal variability. Recent observations $(20 \mathrm{ks}$, PI Kochanek) were also aimed at studying point-source populations, which is beyond the scope of this paper.

\subsection{Immediate objectives}

This paper focuses on a detailed analysis of the extended emission from the hot gas of NGC 6946 with the use of the data acquired by the XMM-Newton X-ray telescope (Jansen et al. 2001). The parameters of the hot gas acquired from the spectral analysis of selected regions of the galaxy are compared with the properties of the radio emission, especially with its polarized component that traces the structure of the magnetic field of the galaxy.

In the following section (Sect. 2) details of the data reduction and analysis are presented. Section 3 presents the distribution of the X-ray emission from both diffuse gas and point-source populations, and we also describe the spectra we obtained. In Sect. 4 we thoroughly discuss the results, including correlations and comparisons with the polarized radio emission from NGC 6946. We also provide new insight into the nature of the ultra-luminous source MF16. We conclude in Sect. 5. 


\section{Observations and data reduction}

NGC 6946 has been observed 11 times between 2003 and 2006 with the XMM-Newton telescope (Jansen et al. 2001), but the observations always suffered from heavy high flaring radiation. The only observation that yielded any clean data was made on 13 June 2004 (ObsID 0200670301). Consequently, the galaxy was observed again, on 2 and 8 November 2007 (ObsIDs 0500730201 and 0500730101, respectively, see Table 2). The data were still affected by high flaring radiation, but this time it was possible to obtain many good-quality data. The effect of frequent flaring radiation on the observations was caused by visibility constraints that required NGC 6946 to be observed at the end of an XMM-Newton revolution. In recent years the orbit has evolved and NGC 6946 can be much better observed. Since early 2012 it has become possible to observe this galaxy for almost a full orbit $(144 \mathrm{ks})$. The most recent observations, performed between 21 and 23 of December 2012 and aimed at the ultraluminous source NGC 6946 X-1 (ObsID 0691570101), provided a long exposure that was relatively free of high flaring radiation; this resulted in $98 \mathrm{ks}$ of good data.

The data were processed using the SAS 13.0.0 package (Gabriel et al. 2004) with standard reduction procedures. Following the routine of tasks epchain and emchain, event lists for two EPIC-MOS cameras (Turner et al. 2001) and the EPICpn camera (Strüder et al. 2001) were obtained. Next, the event lists were carefully filtered for periods of intense radiation of high-energy background by creating light curves of high-energy emission. These light curves were used to produce good time interval (GTI) tables, which mark times of low count rates of high-energy emission. Such tables (time ranges) were then used to remove the remaining data when high count rates were observed. The resulting lists were checked for the residual existence of soft proton flare contamination, which could influence the faint extended emission. To do that, we used a script ${ }^{1}$ that performs calculations developed by Molendi et al. (2004). We found that only the shortest observation (ObsID 0200670301) is contaminated very slightly by soft proton radiation. To ensure the best-quality data (crucial to analyse diffuse emission), we only used events with FLAG $=0$ and PATTERN $\leq 4$ (EPIC-pn) or FLAG $=0$ and PATTERN $\leq 12$ (EPIC-MOS) in the following data processing.

The filtered event lists were used to produce images, background images, exposure maps (without vignetting correction), masked for an acceptable detector area using the images script ${ }^{2}$, modified by the authors to allow adaptive smoothing. All images and maps were produced (with exposure correction) in four energy bands of $0.2-1 \mathrm{keV}, 1-2 \mathrm{keV}, 2-4.5 \mathrm{keV}$, and $4.5-12 \mathrm{keV}$. The images were then combined into final EPIC images and adaptively smoothed with a maximum smoothing scale of $30^{\prime \prime}$ FWHM. The rms values were obtained by averaging the emission over a large source-free area in the final map.

Another set of images was also constructed after excluding all point sources found within the $\mathrm{D}_{25}$ diameter of NGC 6946 from the event lists (see below for details on point source exclusion). This was done with the help of a routine used to create re-filled blank sky background maps - ghostholes_ind ${ }^{3}$. In this way we obtained a map of diffuse emission where all regions of

\footnotetext{
1 http://xmm2.esac.esa.int/external/xmm_sw_cal/ background/epic_scripts.shtml\#flare

2 http://xmm.esac.esa.int/external/xmm_science/ gallery/utils/images.shtml

3 ftp://xmm.esac.esa.int/pub/ccf/constituents/extras/ background/epic/blank_sky/scripts
}

excluded point sources are filled with emission close to extracted regions by sampling adjacent events and randomising spatial coordinates ${ }^{4}$. Although this method is used to handle background maps, we obtained good results when we applied it to real source data. Section 3.1.1 presents images of soft $(0.2-1 \mathrm{keV})$ and medium $(1-2 \mathrm{keV})$ emission, together with a corresponding hardness ratio map, defined as

$H R=\frac{\text { med }-\mathrm{soft}}{\text { med }+ \text { soft }}$,

for images with and without detected point sources.

Next, the spectral analysis was performed. To create spectra we only used the event list from the EPIC-pn camera because it offers the highest sensitivity in the soft energy band. Only the emission above $0.3 \mathrm{keV}$ was analysed because the internal noise of the pn camera is too high below this limit ${ }^{5}$. Although this is not crucial when combined with MOS cameras to produce images, it is important to exclude the softest emission below $0.3 \mathrm{keV}$ to obtain reliable good-quality spectra.

Unsmoothed images for all bands were used to search for point sources with the standard SAS edetect_chain procedure. Regions found to include a possible point source were marked. The area was individually chosen for each source to ensure that we excluded all pixels brighter than the surrounding background. These areas were then used to construct spectral regions for which spectra were acquired.

The non-default way of excluding the detected point sources helped to keep more diffuse emission in the final spectra. However, expecting some contribution from the PSF wings, we added a power-law component to our model fits to account for any residual emission. A power-law component was also needed to account for unresolved point sources. The background spectra were obtained using blank sky event lists (see Carter \& Read 2007). These blank sky event lists were filtered using the same procedures as for the source event lists. For each spectrum we produced response matrices and effective area files. For the latter, detector maps needed for extended emission analysis were also created. The spectra were binned, which resulted in a better signal-to-noise ratio. To obtain a reasonable number of bins at the same time, we chose to have 25 total counts per energy bin. The spectra were fitted using XSPEC 11 (Arnaud 1996).

Since observations 0500730201 and 0500730101 have identical pointings and position angles, we merged the cleaned event lists using the SAS task merge. The spectra extracted from this merged lists give the same model-fitting results as separate spectra fitted simultaneously, but the errors are better constrained, therefore we used the former spectra in our final analysis. It was not possible to also merge-in the shortest observation (ObsID 0200670301) because it has different parameters (pointing and position angle), and simultaneous fitting with the larger data set showed that both spectra are systematically offset. This resulted in a poorer model fit. We assume that this might arise because the observation was significantly affected by high flaring radiation and because the filtered "clean" data still show residual contamination that might influence the spectral fitting. Although we used this observation for image production, we therefore excluded the pn data from our spectral analysis. For the same inconsistency reasons (different pointing and position angle), we used the longest observation 0691570101 separately

\footnotetext{
4 http://xmm2.esac.esa.int/external/xmm_sw_cal/ background/blank_sky.shtml\#BGsoft

5 http://xmm.esac.esa.int/external/xmm_user_support/ documentation/uhb
} 

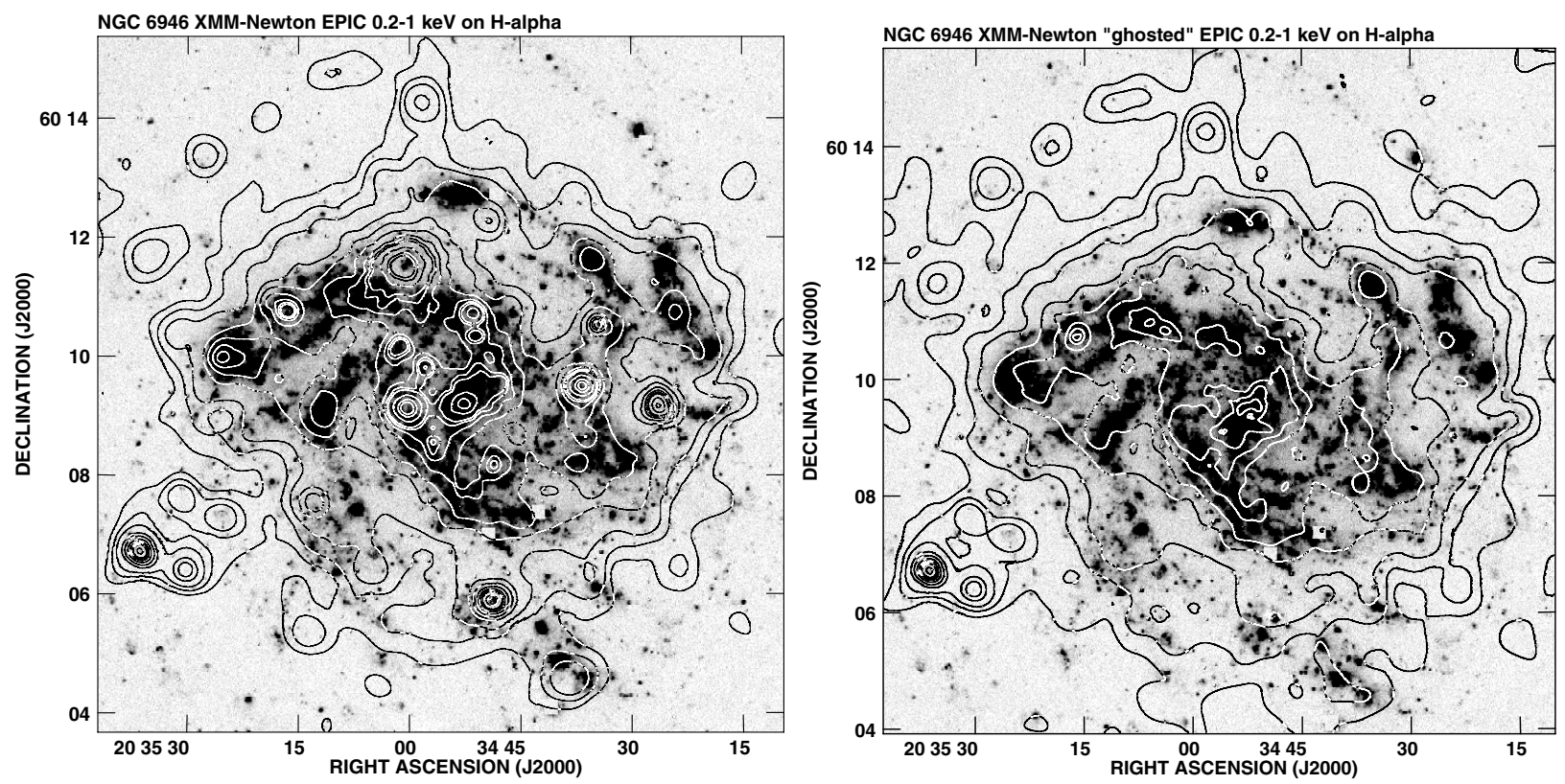

Fig. 1. Left: map of soft X-ray emission from NGC 6946 in the $0.2-1 \mathrm{keV}$ band overlaid onto an $\mathrm{H} \alpha$ image. The contours are 3, 5, 8, 16, 25, 40, $60,80,100,200,500$, and $1000 \times \mathrm{rms}$. The map is adaptively smoothed with the largest scale of $30^{\prime \prime}$. Right: same map, but with point sources excluded from the galactic disk (see text for details).

when performing the spectral analysis. This approach resulted in two corresponding spectra for each of the studied regions. Each pair of spectra was merged (as well as their corresponding background spectra) using the SAS task epicspeccombine. Although for multiple spectra a most commonly advised routine is a simultaneous fit rather than a fit to a combined spectrum, we note that for spectra with a very different sensitivity (certainly in our case), a combination of spectra leads to a much better handle on the background and consequently a better fit. This is because background subtraction only takes place for the merged spectrum after the source and background spectra are combined (contrary to a simultaneous fit, where each spectrum is background subtracted before the fit).

For the overlays we also used the XMM-Newton Optical Monitor data acquired during the same observations and produced an image in the UVM2 filter using the standard SAS omchain procedure.

\section{Results}

\subsection{Distribution of the $X$-ray emission}

\subsubsection{Diffuse emission}

NGC 6946 shows soft extended X-ray emission corresponding to the entire star-forming disk (Fig. 1), with the brightest emission closely following the star-forming regions. Although the southern part of the star-forming disk is less pronounced than the northern one, no asymmetries of the emission from the hot gas are visible. In contrast, the X-ray emission seems to extend farther out beyond star-forming regions in the southern part of the galaxy. An area of diffuse emission around the galactic centre forms a structure resembling a very small bar that crosses the central core and is aligned with the $\mathrm{H} \alpha$ emission.

The hot gas disk visible in the $1-2 \mathrm{keV}$ energy band (Fig. 2) is extended in a similar way as the emission in the softer energy band (Fig. 1). This may suggest large amounts of very hot gas in the galactic disk and/or halo. To further investigate the contribution from the hottest gas to the X-ray emission from
NGC 6946, we produced a hardness ratio map using both distributions (Fig. 3).

As mentioned before, the two distribution are similar on average because the values in most parts of the HR map are close to 0 . Nevertheless, north-east and south-west of the centre, distinct areas of softer emission are clearly visible. This corresponds well to the orientation of the bright star-forming regions visible in the $\mathrm{H} \alpha$ map (Fig. 1). However, the softest emission is produced in the south-western part of the disk, where the production of young massive stars is diminished, as seen in the UV map (Fig. 2).

\subsubsection{Point sources}

Figure 4 shows all detected point sources within the $\mathrm{D}_{25}$ disk of NGC 6946. For all sources we performed a spectral analysis. For weak sources the hardness ratios (HRs) were derived (see Table 3). We used the same energy bands as in Pietsch et al. (2004): (0.2-0.5) keV, (0.5-1.0) keV, (1.0-2.0) keV, $(2.0-4.5) \mathrm{keV}$, and $(4.5-12) \mathrm{keV}$ as bands 1 to 5 . Consequently, the hardness ratios are calculated as $H R_{i}=B_{i+1}-B_{i} / B_{i+1}+B_{i}$ for $i=1$ to 4 , where $B_{i}$ is the count rate in band $i$, as defined above. For sources with more than 500 net counts in the total energy band $(0.2-12 \mathrm{keV})$, spectra were extracted and fitted with models (see Sect. 3.2.1).

The left panel of Fig. 4 suggests that some of the sources might not originate from within the galaxy and are instead more distant background objects. Moreover, the centre of the most extended region of source 26 does not seem to coincide with the visible $\mathrm{H} \alpha$ clump, but more precise astrometry of the Chandra observation associated this source with the galaxy (e.g. Kajava $\&$ Poutanen 2009). This source is the brightest point source the ultra-luminous complex MF16 that is assumed to be a supernova remnant (e.g. Matonick \& Fesen 1997). It is not a pure point source, as it is considerably larger than the point spread function of the XMM-Newton EPIC cameras (of $\simeq 12^{\prime \prime}$ ). 

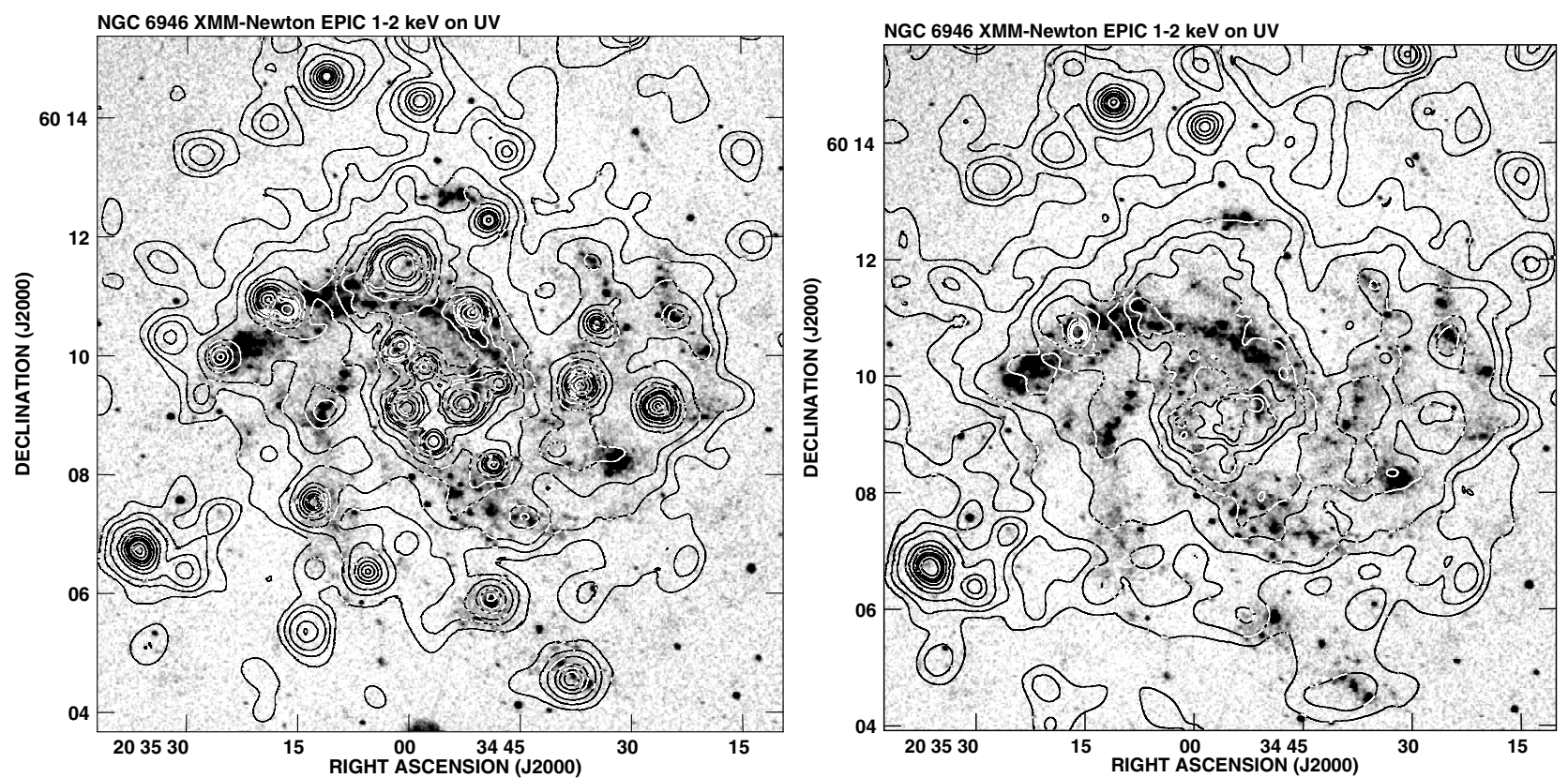

Fig. 2. Left: map of medium X-ray emission from NGC 6946 in the 1-2 keV band overlaid onto the XMM-Newton Optical Monitor UVM2 filter image. The contours are 3, 5, 8, 16, 25, 40,60, 80, 100, 200,300, 500, and $1000 \times$ rms. The map is adaptively smoothed with the largest scale of $30^{\prime \prime}$. Right: same map, but with point sources excluded from the galactic disk (see text for details).
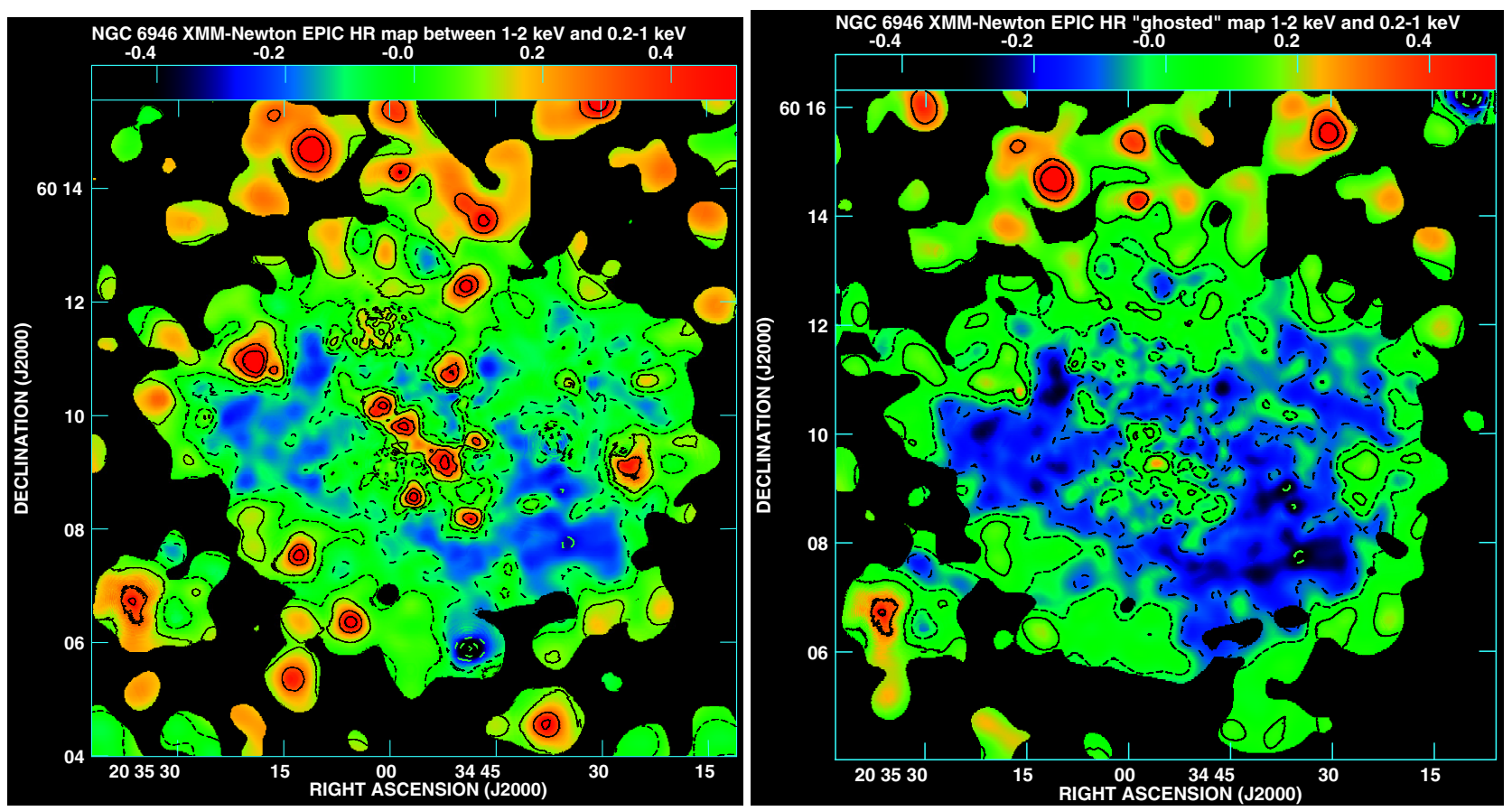

Fig. 3. Maps of the hardness ratio between medium and soft X-ray emission from NGC 6946 (Figs. 2 and 1). The map is truncated at the $3 \sigma$ level of the 1-2 keV map. Left: map with point sources. Right: map without point sources.

Another interesting source is source 17, overlapping in Fig. 4 with sources 16 and 20 . This source was not visible in previous observations (where sources 16 and 20 were detected), but only in the most recent sensitive observations.

\subsection{Spectral analysis of the $X$-ray emission}

For spectra of the hot gas we used a model that attributed one or two thermal plasmas and/or a contribution from unresolved point-like sources. Thermal plasma is represented in this work by a mekal model, which is a model of an emission spectrum from hot diffuse gas based on the model calculations of Mewe and Kaastra (Mewe et al. 1985; Kaastra 1992). In all models we fixed the metallicity to solar. This was determined by a test spectral fitting of the spectra, which showed that in a wide range of metallicities (0.1-1.5 solar), no significant change in gas temperatures or power-law photon indices could be observed. Slight deviations of these parameters were still within the errors provided by the models that used solar metallicity. This 


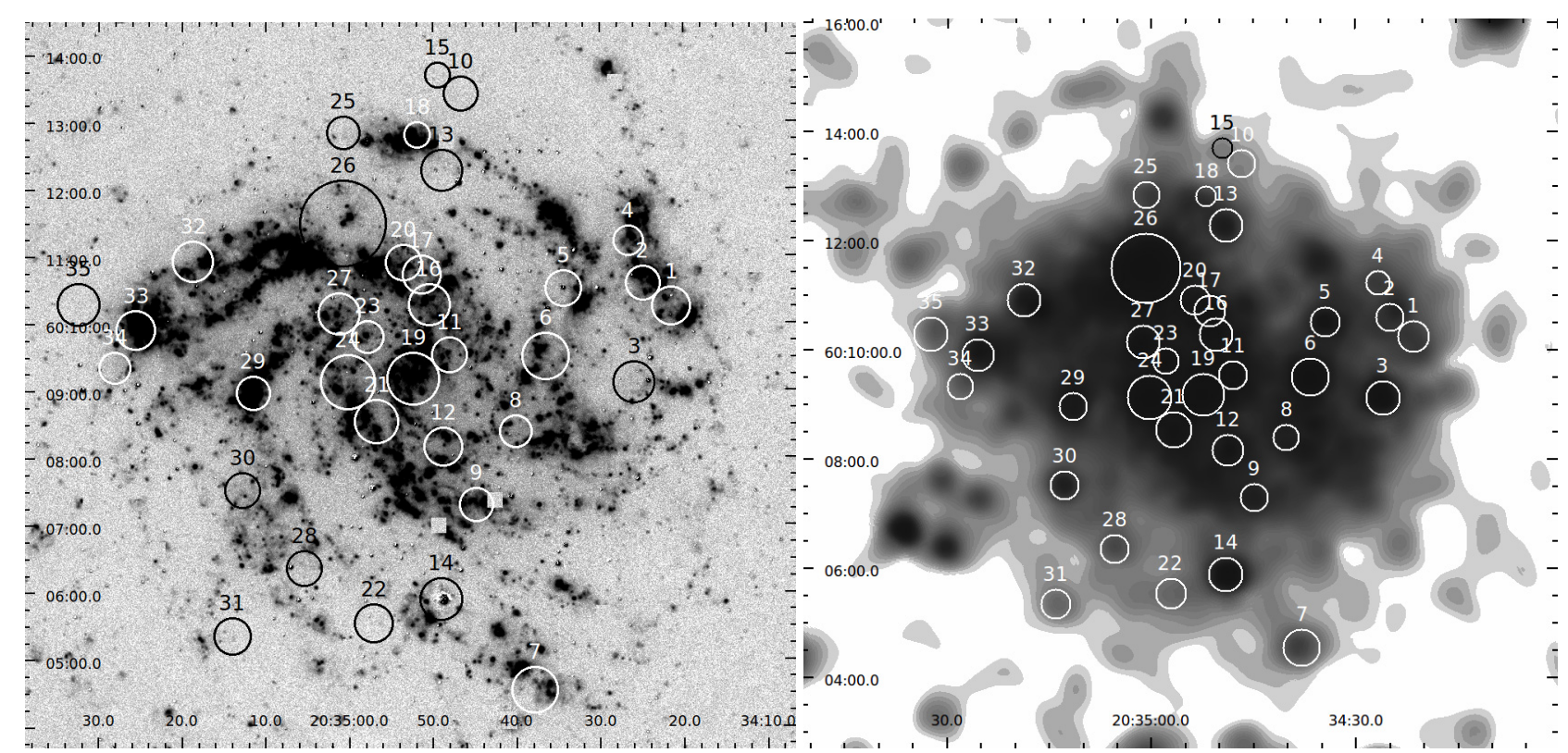

Fig. 4. Left: all point-like sources detected in NGC 6946 (see text for a detailed description) overlaid on an H $\alpha$ image. Right: the same regions as in the left panel overlaid on the map of soft X-ray emission in the $0.2-1 \mathrm{keV}$ band as shown in Fig. 1.

suggests that all differences that are found in gas temperatures when fitting spectra do not result from a possible combined effect of abundance gradients and enforcing solar metallicities in the models. It also shows that an additional power-law component in the models of diffuse emission does account for emission from unresolved point-like sources and is not introduced to mimic variable gas abundance because excluding the powerlaw component from the model and again varying metallicities resulted in unphysical values of the parameters. Consequently, the quality of the model fits remained very low (with reduced $\left.\chi^{2}>2\right)$.

A contribution from unresolved point-like sources is fitted with a simple power law. For some models an additional component to account for the internal absorption needed to be used. For all models we also used a fixed foreground (galactic) absorption. Tables 4 and 8 present an overview of the various spectral models, including the obtained parameters. The errors provided for the model parameters are always $1 \sigma$ errors. The derived X-ray fluxes and luminosities are shown in Tables 5 and 9.

The same model components were used for the spectral analysis of the brightest point sources (see Sect. 3.1.2). Since we did not aim to perform a variability analysis of point sources, we used all data that were suitable for spectral analysis (for the selection and merging of separate data sets, see Sect. 2), similarly as for regions of diffuse emission.

The regions of the diffuse X-ray emission from which the spectra were extracted are presented in Fig. 9. Extraction areas for the brightest point-sources are the same as found by the source-detection analysis (cf. Fig. 4). All plots of the modelled spectra together with the fitted models are presented in Figs. 11 and 12 .

\subsubsection{Brightest sources}

For almost all sources (except for source 27) for which spectral fitting was performed, we also used the thermal plasma component (see Table 4) to account for diffuse emission projected onto each of the sources. The amount of this emission was often significant as a result of the sensitive observations of a gas-rich galaxy with a relatively large point spread function of the EPIC-pn camera that did not allow clearly separating the studied sources. Many of the spectra also required an additional model component to account for the high internal absorption.

For one source (source 5) a model with a power-law component did not give good results. The spectrum was fitted best when using two thermal components. It is possible that it is simply a hot gas clump that was classified by the source detection routine to be a point source. Alternatively, the lowest count number of all fitted spectra might also be the reason for difficulties in finding a proper fit. The hot component fitted in this model reaches values of almost $2 \mathrm{keV}$, which is fairly unexpected for the hot gas in the galactic disk.

For source 17, which was undetected in earlier observations, a complex model had to be fitted. Its power-law component is poorly constrained, however. The two sources near this position that were detected previously, sources 16 and 20, were not detected in the recent sensitive observation because the brightness of the diffuse emission was higher throughout, and also because the bright source 17 has wings. For consistency of the results, however, we used all observations to extract spectra for all three sources. Nevertheless, the complexity of the model for source 17 might be caused by admixtures from the two adjacent sources.

The spectrum of the core region, source 19, where the densest and the hottest gas might be expected, could be fitted equally well with either one or two thermal components. We present both model fits. The model with two components yielded $0.32_{-0.03}^{+0.02} \mathrm{keV}$ and $0.70_{-0.06}^{+0.07} \mathrm{keV}$, while a single temperature component showed a temperature of $0.62 \pm 0.04 \mathrm{keV}$. These values and the derived fluxes for this region (see Table 5) suggest that most of the emission originates from the hotter component. The main difference between both model fits, however, is a significant variation of the internal absorption (see Table 4).

By fitting a model (consisting of one power-law and two thermal components) to the spectrum of MF16 (source 26), we obtained a temperature of $1.04 \pm 0.03 \mathrm{keV}$ for the hot component. Still, most of the flux from MF16 originates from the power-law component that is associated with the central source. To further investigate the spectrum of this source, we followed 


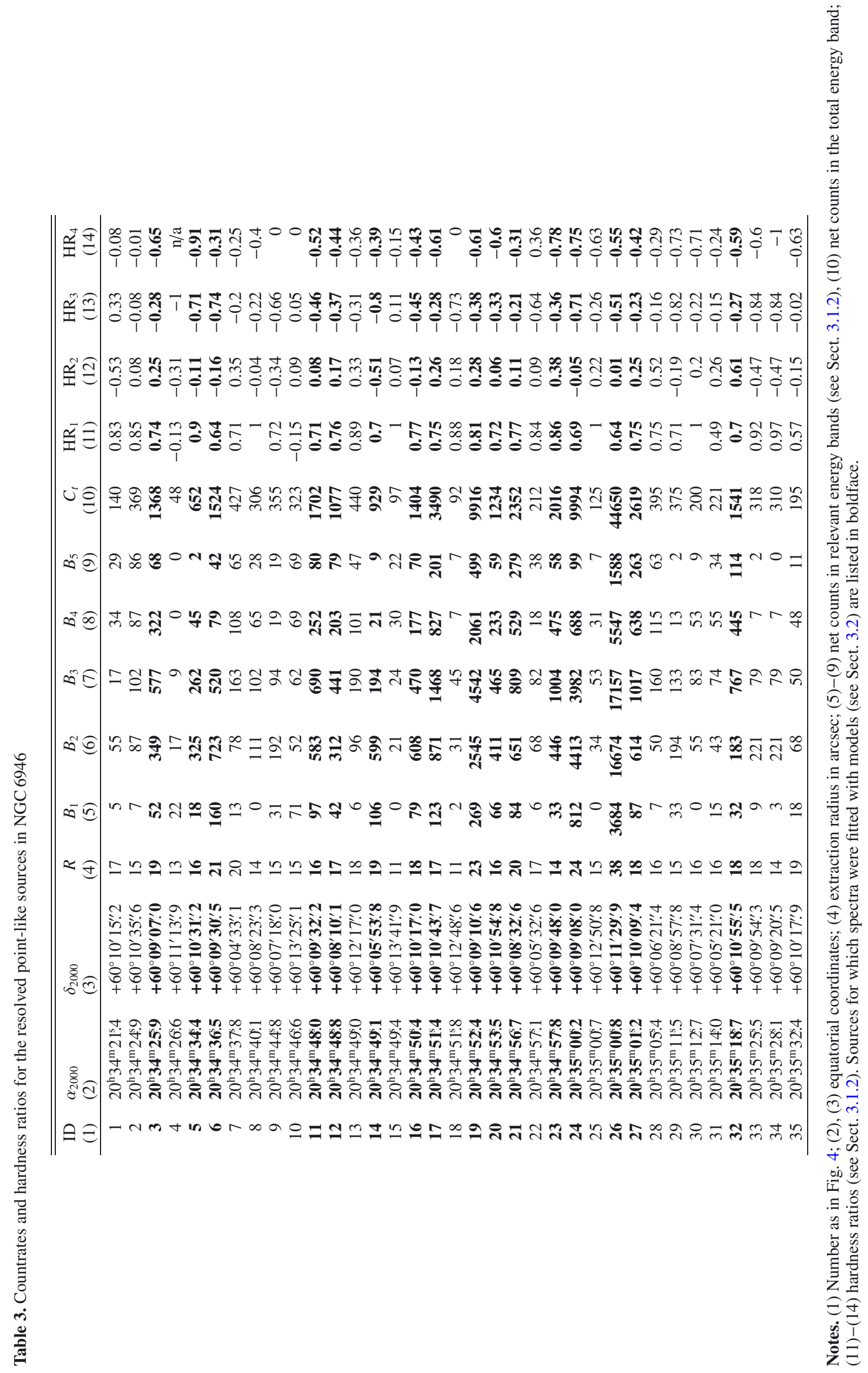


A\&A 585, A3 (2016)
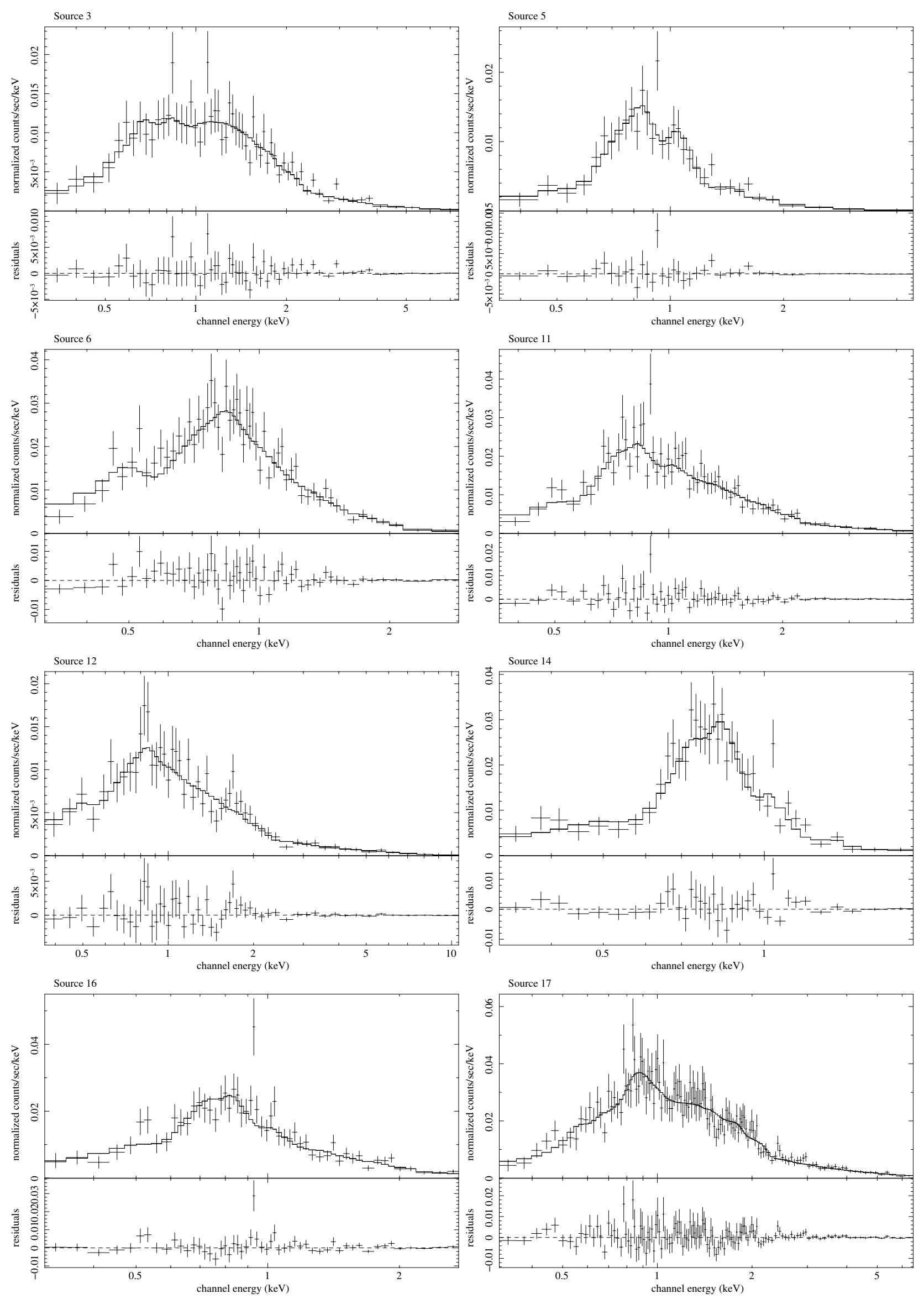

Fig. 5. Model fits to the spectra of selected sources in NGC 6946. See text and Tables 4 and 5. 
Table 4. Model-fit parameters for the point sources studied in NGC 6946.

\begin{tabular}{|c|c|c|c|c|c|c|c|}
\hline ID & $\begin{array}{l}\text { Model } \\
\text { type }\end{array}$ & $\begin{array}{c}\text { Internal } \\
\mathrm{nH}^{a}\end{array}$ & $\begin{array}{c}k T_{1} \\
{[\mathrm{keV}]}\end{array}$ & $\begin{array}{c}k T_{2} \\
{[\mathrm{keV}]}\end{array}$ & $\begin{array}{l}\text { Photon } \\
\text { Index }\end{array}$ & $\chi_{\text {red }}^{2}$ & $\begin{array}{r}\text { Net } \\
\text { counts }\end{array}$ \\
\hline 3 & wabs(mekal+wabs*power law) & $4.19_{-1.76}^{+2.07}$ & $0.23_{-0.05}^{+0.04}$ & - & $2.62 \pm 0.23$ & 0.94 & 1368 \\
\hline 5 & wabs(mekal+mekal) & - & $0.54 \pm 0.09$ & $1.93_{-0.32}^{+0.65}$ & - & 0.80 & 652 \\
\hline 6 & wabs(mekal+wabs*power law) & $1.86_{-0.44}^{+0.47}$ & $0.60_{-0.35}^{+0.11}$ & - & $3.91_{-0.41}^{+0.61}$ & 1.04 & 1524 \\
\hline 11 & wabs(mekal+wabs*power law) & $1.78_{-0.54}^{+1.66}$ & $0.38_{-0.08}^{+0.16}$ & - & $2.48_{-0.21}^{+0.48}$ & 0.99 & 1702 \\
\hline 12 & wabs(mekal+power law) & - & $0.58_{-0.22}^{+0.21}$ & - & $1.78 \pm 0.12$ & 0.91 & 1077 \\
\hline 14 & wabs(mekal+power law) & - & $0.46_{-0.08}^{+0.06}$ & - & $3.12_{-0.91}^{+0.56}$ & 1.12 & 929 \\
\hline 16 & wabs(mekal+power law) & - & $0.38_{-0.06}^{+0.21}$ & - & $2.03_{-0.13}^{+0.15}$ & 1.15 & 1404 \\
\hline 17 & wabs(mekal+mekal+wabs*power law) & $5.29_{-1.33}^{+2.05}$ & $0.18 \pm 0.02$ & $0.66 \pm 0.06$ & $2.31_{-1.04}^{+0.83}$ & 0.91 & 3490 \\
\hline 19 & wabs(mekal+wabs(mekal+power law)) & $6.87_{-1.21}^{+1.02}$ & $0.32_{-0.03}^{+0.02}$ & $0.70_{-0.06}^{+0.07}$ & $2.55_{-0.03}^{+0.07}$ & 1.02 & 9916 \\
\hline $19^{b}$ & wabs(mekal+wabs*power law) & $2.89_{-0.33}^{+0.31}$ & $0.62 \pm 0.04$ & - & $2.36_{-0.07}^{+0.03}$ & 1.03 & 9916 \\
\hline 20 & wabs(mekal+power law) & - & $0.62_{-0.12}^{+0.08}$ & - & $1.65_{-0.11}^{+0.12}$ & 0.90 & 1234 \\
\hline 21 & wabs(mekal+power law) & - & $0.45_{-0.06}^{+0.08}$ & - & $1.52_{-0.07}^{+0.08}$ & 1.12 & 2352 \\
\hline 23 & wabs(mekal+wabs*power law) & $3.29_{-1.05}^{+0.81}$ & $0.30_{-0.07}^{+0.21}$ & - & $2.52_{-0.20}^{+0.22}$ & 1.10 & 2016 \\
\hline 24 & wabs(mekal+wabs*power law) & $2.16_{-0.21}^{+0.17}$ & $0.78 \pm 0.05$ & - & $3.80 \pm 0.15$ & 1.23 & 9994 \\
\hline 26 & wabs(mekal+mekal+power law) & - & $0.40_{-0.03}^{+0.09}$ & $1.04 \pm 0.03$ & $2.26 \pm 0.03$ & 1.19 & 44650 \\
\hline $26^{c}$ & wabs(mekal+mekal+power law+diskbb) & - & $0.66 \pm 0.06$ & $1.34_{-0.22}^{+0.32}$ & $1.98 \pm 0.08$ & 1.11 & 44650 \\
\hline 27 & wabs*power law & - & - & - & $1.68 \pm 0.05$ & 1.05 & 2619 \\
\hline 32 & wabs(mekal+wabs*power law) & $8.83_{-1.70}^{+2.15}$ & $0.25_{-0.05}^{+0.06}$ & - & $2.82_{-0.17}^{+0.26}$ & 0.80 & 1541 \\
\hline
\end{tabular}

Notes. ${ }^{(a)}$ Column density in $\left[10^{21} \mathrm{~cm}^{-2}\right] .{ }^{(b)}$ Single temperature model for the thermal component. ${ }^{(c)}$ Model with an additional multicolour disk model component of $T_{\text {in }}=0.26_{-0.01}^{+0.02} \mathrm{keV}$. ID 19 is the core central source in NGC 6946 and ID 26 is MF16.

Kajava \& Poutanen (2009) and added a multicolour disk component to our model. We obtained a similar value for the temperature of the inner disk.

\subsubsection{Regions of diffuse emission}

To analyse emission of the hot gas from NGC 6946, we used $\mathrm{H} \alpha$ and UV images to choose areas that correspond to the starforming regions. The radio morphology of the galaxy was also taken into account because the most prominent polarized features were found between the gaseous spiral arms of NGC 6946 (see Fig. 10). All selected regions are presented in Fig. 9. A brief description of all regions is presented in Table 6. For transparency, region letters are used throughout this paper. In the process of extracting the spectra, the emission from point sources (Fig. 4) was excluded.

To investigate the temperature of the hot gas in selected regions of NGC 6946, we fitted a single thermal plasma model, adding a power-law component to account for undetected point sources and/or residual emission from excluded sources. Only regions $\mathrm{C}$ and $\mathrm{F}$ did not require this additional power-law component. This could simply be caused by a lower number of net counts in their spectra, resulting in a low signal-to-noise ratio, hence lower accuracy of the fitting, so that the basic models are equally good in fitting the data. On the other hand, both spectra show very little emission above $1 \mathrm{keV}$, which suggests that the harder emission from point sources contributes hardly anything.

In Table 7 we present the results of single thermal plasma model fitting. For regions of the spiral galactic arms (regions A,
E, G, and H), very steep photon indices of the power-law component are visible, which is unexpected for typical galactic X-ray point sources. This might be due to enhanced emission in the soft-to-medium energy band (around and above $1 \mathrm{keV}$ ), however, which can also suggest that a second thermal component is necessary to account for the significant emission from the hot gas in the galactic disk. To check this possibility, we fitted a model consisting of two thermal components for the areas of the spiral arms and kept the previously introduced powerlaw component. Since both parameters and residuals of the new fits were much more physical, that is, showed values in expected ranges, we used them as the final fits for the subsequent analysis.

To ensure that a single thermal plasma model is the bestfit model for the remaining regions (central areas and interarm regions), we also fitted a model with two thermal components to their corresponding spectra. In all cases we obtained significantly flatter (lower) photon indices of the power-law component, which suggests that some of the harder emission from the unresolved point sources was fitted with the new second thermal component. Furthermore, most of the fitted parameters were poorly constrained. For some of them it was impossible to get any constraints. The final models for all regions are presented in Table 8.

For regions of the spiral arms for which a model with two thermal components was used, we assumed that the cooler component $(\sim 0.3 \mathrm{keV})$ of the hot gas is associated with the galactic halo and the hotter component $(\sim 0.7 \mathrm{keV})$ with the emission from the disk, as is observed in edge-on galaxies (e.g. Tüllmann et al. 2006). 

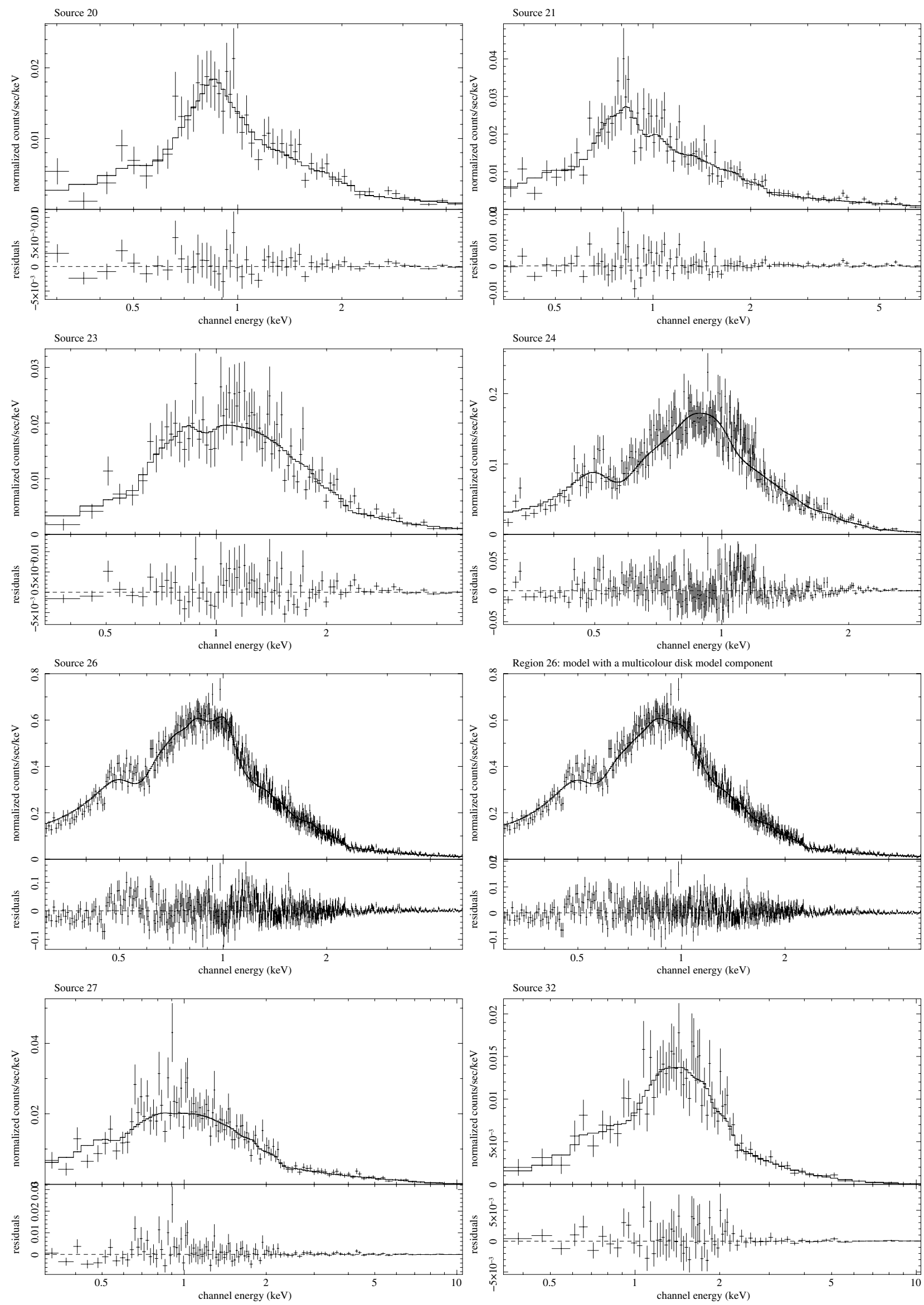

Fig. 6. Model fits to the spectra of selected sources in NGC 6946. See text and Tables 4 and 5. 

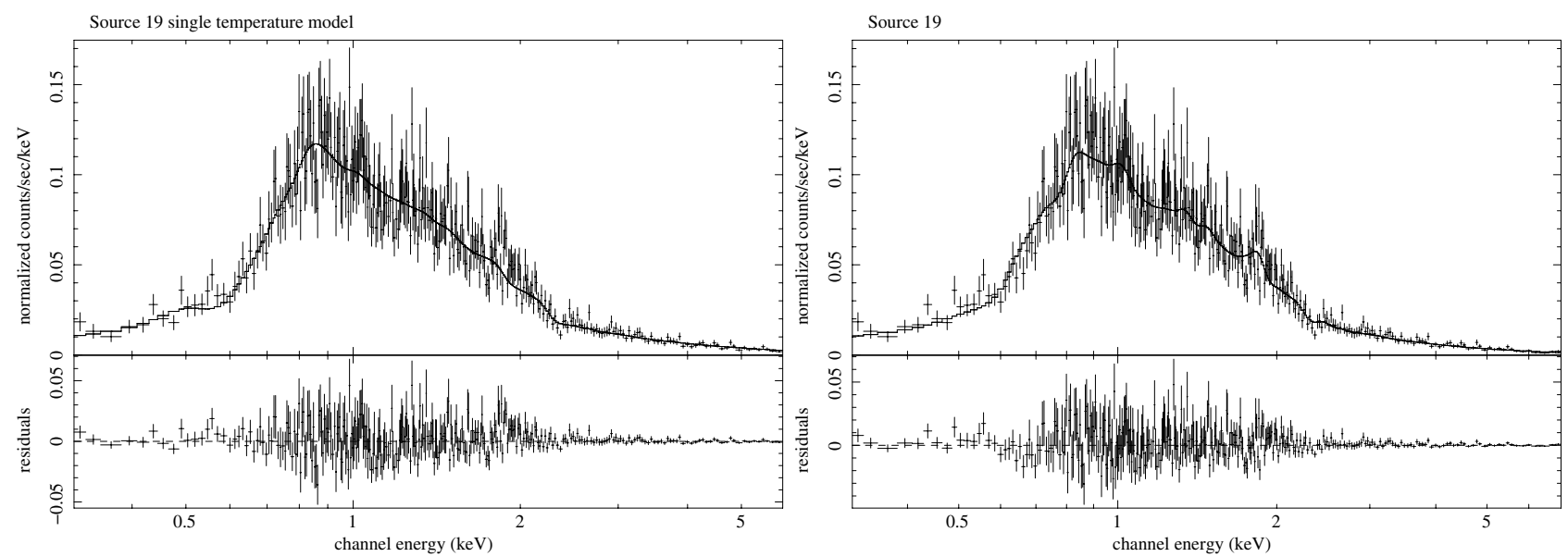

Fig. 7. Model fits to the spectra of the nuclear region of NGC 6946. See text and Tables 4 and 5.
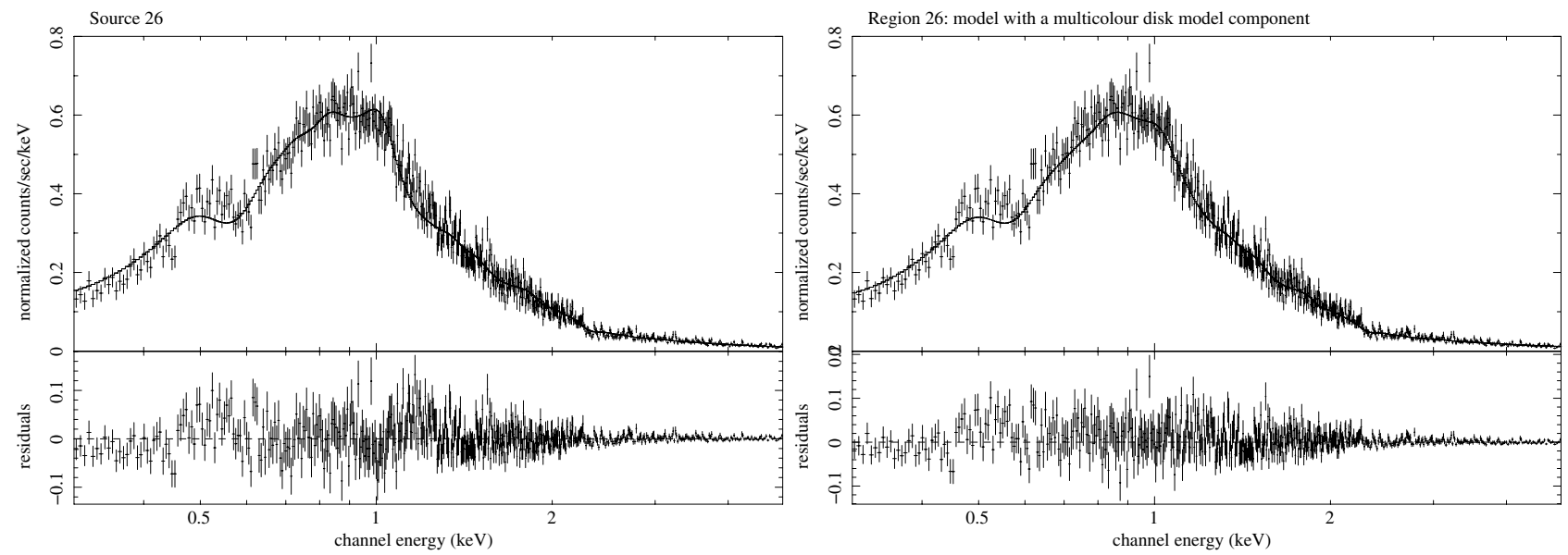

Fig. 8. Model fits to the spectra of MF16. See text and Tables 4 and 5.

For the remaining regions, that is, for the central areas and the interarm regions, we assumed that the single thermal component can be described as a mixture of gas components from the disk and the halo above. The parameters of these components seem to be similar to the level, which does not allow clearly separating them at a given sensitivity level. For the interarm regions this can be easily explained by the lack of starforming regions in the disk, which causes the gas to be relatively uniform throughout the entire volume. Nevertheless, we cannot use this argument for the central regions of the galaxy, where the disk emission is certainly significant. A reasonable explanation for this case is a high star-forming activity of NGC 6946 and the consequent internal absorption that is highest in the central densest part of the galactic disk. This absorption may cause some of the disk emission from the central regions I and $\mathrm{J}$ to remain hidden, and the rest, together with the halo gas, mimics a homogenous medium.

Region B shows almost no $\mathrm{H} \alpha$ or UV emission, while region $\mathrm{J}$ includes the central parts of the galaxy. Both regions differ significantly in the value of the fitted temperature of the hot gas. It is much hotter $\left(0.60_{-0.08}^{+0.06} \mathrm{keV}\right)$ for the quiet region $\mathrm{B}$ than for region $\mathrm{J}$ with its clear galactic-disk (nuclear) emission $\left(0.42_{-0.05}^{+0.09} \mathrm{keV}\right)$. The remaining single thermal component model fits show that the temperature of the hot gas is between $0.5 \mathrm{keV}$ and $0.55 \mathrm{keV}$. Halo components of the model fits to the regions of spiral arms show a very constant temperature of around $0.28 \mathrm{keV}$. For the disk components, however, differences are observed, as the south-western and western part of the disk (spiral arms $\mathrm{A}$ and $\mathrm{H}$ ) have a significantly lower temperature than the eastern and north-eastern part (spiral arms E and $\mathrm{G})$.

For all regions except for region $\mathrm{H}$ (western spiral arm) most of the X-ray flux is produced by the power-law component that is attributed to unresolved and/or residual emission from point sources. For the exceptional region $\mathrm{H}$ we note, however, that the value of the power-law flux is only poorly constrained. Therefore, its value might be much higher, which would lead to a higher contribution to the total flux. A high contribution from the power-law components of the fits might arise because NGC 6946 is a starburst galaxy with a large population of X-ray point sources (Fig. 4). The method of source extraction used in this paper, described in Sect. 2, might certainly cause this effect. However, as argued before, this ensured that as little as possible of the diffuse emission has been lost by point-source extraction.

For the regions of the spiral arms, more flux comes from the halo components according to the fits, which agrees with a physical picture where halo emission is produced in a much larger volume density of the underlying star formation. These fractions are equal only for the western spiral arm (region $\mathrm{H}$ ); we discuss this in Sect. 4.2.1 in more detail. 
Table 5. Total $(0.3-12 \mathrm{keV})$ unabsorbed fluxes in $10^{-14} \mathrm{erg} \mathrm{cm}^{-2} \mathrm{~s}^{-1}$ for modelled point sources in NGC 6946 along with luminosities of their power-law components in $10^{39} \mathrm{erg} \mathrm{s}^{-1}$.

\begin{tabular}{|c|c|c|c|c|c|}
\hline ID & Mekal cold & Mekal hot & Power law & Total & Luminosity \\
\hline 3 & $1.5_{-0.8}^{+0.6}$ & - & $18.8_{-4.3}^{+6.3}$ & $20.3_{-5.1}^{+6.8}$ & $1.10_{-0.25}^{+0.37}$ \\
\hline 5 & $1.0_{-0.2}^{+0.1}$ & $1.1_{-0.2}^{+0.3}$ & - & $2.1 \pm 0.4$ & - \\
\hline 6 & $2.0_{-1.1}^{+0.7}$ & - & $41.1_{-14.0}^{+31.3}$ & $43.1_{-15.2}^{+32.0}$ & $2.41_{-0.82}^{+1.84}$ \\
\hline 11 & $0.8_{-0.4}^{+0.9}$ & - & $9.7_{-2.5}^{+4.9}$ & $10.5_{-2.9}^{+5.8}$ & $0.57_{-0.15}^{+0.29}$ \\
\hline 12 & $0.3 \pm 0.2$ & - & $5.3_{-1.2}^{+1.3}$ & $5.6_{-1.4}^{+1.5}$ & $0.31_{-0.07}^{+0.08}$ \\
\hline 14 & $2.0_{-0.4}^{+0.8}$ & - & $1.3_{-0.6}^{+1.0}$ & $3.3_{-0.7}^{+1.1}$ & $0.08_{-0.04}^{+0.06}$ \\
\hline 16 & $1.2_{-0.4}^{+0.5}$ & - & $4.3_{-0.8}^{+1.0}$ & $5.5_{-1.3}^{+1.6}$ & $0.25_{-0.05}^{+0.06}$ \\
\hline 17 & $1.6_{-0.5}^{+0.6}$ & $1.6 \pm 0.6$ & $25.7_{-5.8}^{+5.2}$ & $28.9_{-6.9}^{+6.3}$ & $1.51_{-0.34}^{+0.30}$ \\
\hline 19 & $4.9_{-0.8}^{+0.6}$ & $26.0_{-11.2}^{+15.6}$ & $69.6_{-2.9}^{+6.5}$ & $100.5_{-14.9}^{+22.8}$ & $4.08_{-0.17}^{+0.38}$ \\
\hline $19^{a}$ & $3.6 \pm 0.6$ & - & $58.6_{-2.4}^{+5.7}$ & $62.2_{-3.0}^{+6.3}$ & $3.44_{-0.14}^{+0.33}$ \\
\hline 20 & $0.8 \pm 0.2$ & - & $5.6_{-1.4}^{+1.6}$ & $6.4_{-1.5}^{+1.7}$ & $0.33_{-0.08}^{+0.09}$ \\
\hline 21 & $0.9 \pm 0.3$ & - & $11.4_{-1.9}^{+2.1}$ & $12.4_{-2.2}^{+2.3}$ & $0.67_{-0.11}^{+0.12}$ \\
\hline 23 & $0.6_{-0.4}^{+0.7}$ & - & $17.7_{-3.3}^{+5.3}$ & $18.3_{-3.8}^{+5.9}$ & $1.04_{-0.19}^{+0.31}$ \\
\hline 24 & $4.5 \pm 0.9$ & - & $108.0_{-14.9}^{+16.7}$ & $112.6_{-15.9}^{+17.6}$ & $6.33_{-0.87}^{+0.98}$ \\
\hline 26 & $10.5_{-2.0}^{+2.5}$ & $14.5_{-1.6}^{+1.7}$ & $128.3_{-4.4}^{+4.2}$ & $153.3_{-8.0}^{+8.4}$ & $7.52_{-0.26}^{+0.25}$ \\
\hline $26^{b}$ & $10.2 \pm 2.4$ & $9.4_{-1.4}^{+2.3}$ & $104.1_{-16.9}^{+14.4}$ & $156.2_{-21.2}^{+32.1}$ & $8.03_{-1.76}^{+2.05}$ \\
\hline 27 & - & - & $14.8_{-1.3}^{+1.4}$ & $14.8_{-1.3}^{+1.4}$ & $0.87^{ \pm} 0.08$ \\
\hline 32 & $0.7 \pm 0.3$ & - & $36.3_{-6.0}^{+9.3}$ & $37.0_{-6.2}^{+9.6}$ & $2.13_{-0.35}^{+0.55}$ \\
\hline
\end{tabular}

Notes. ${ }^{(a)}$ Single temperature model for the thermal component. ${ }^{(b)}$ Model with an additional multicolour disk model component with a flux of $32.9_{-12.8}^{+21.4} \times 10^{-14} \mathrm{erg} \mathrm{cm}^{-2} \mathrm{~s}^{-1}$. ID 19 is the core central source in NGC 6946 and ID 26 is MF16.

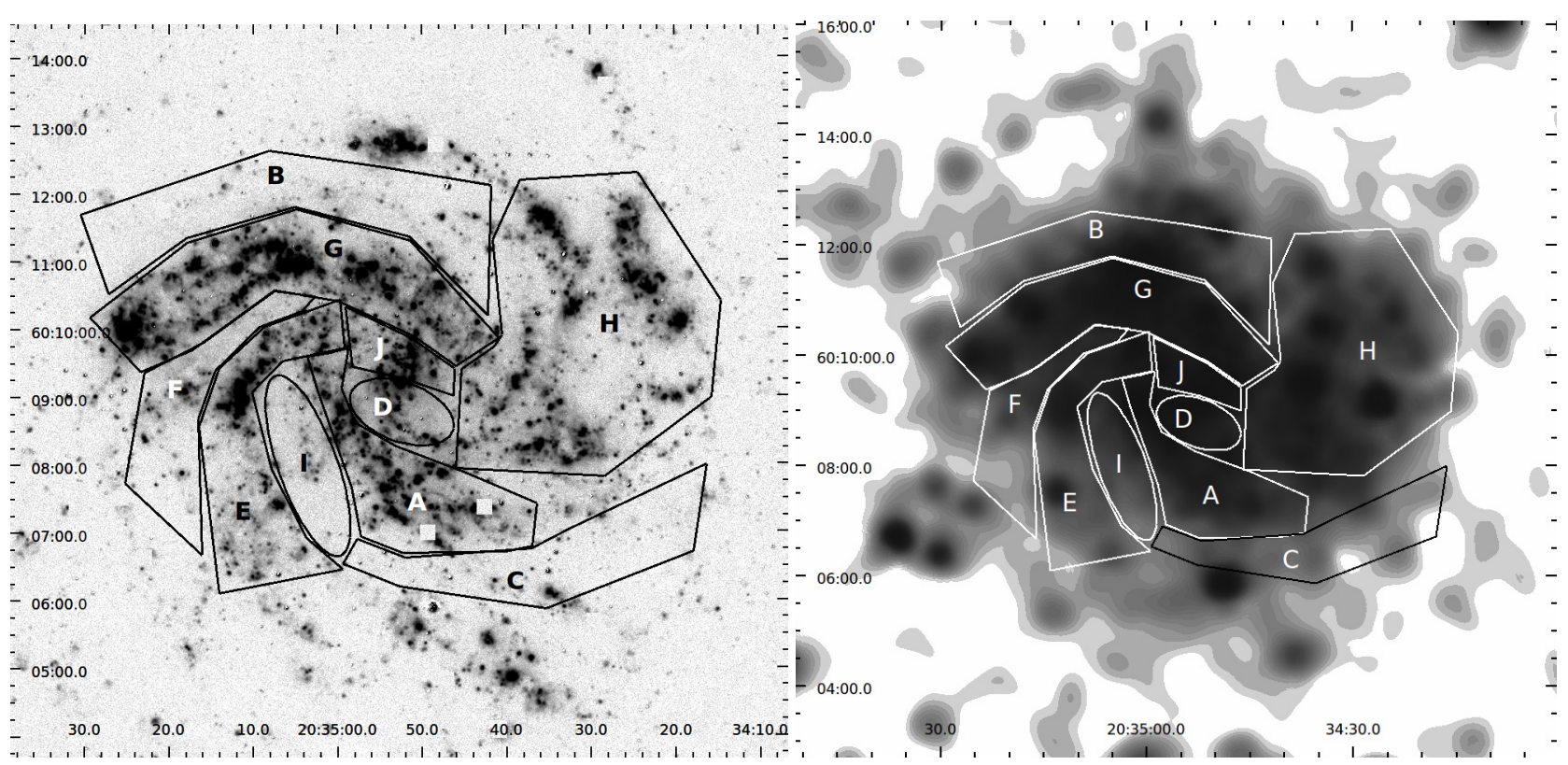

Fig. 9. Regions of diffuse X-ray emission from NGC 6946 (see text for a detailed description) overlaid on the same maps as in Fig. 4.

\section{Discussion}

\subsection{Point sources}

Of the 35 point sources found in the galactic disk of NGC 6946, for 19 of them we were able to calculate only the hardness ratios. All values are presented in Table 3. For the remaining sources we fitted models to the acquired spectra (see Sect. 3.2.1). As mentioned above, all sources except for source 27 required an additional thermal component in the model. This was most likely due to the characteristics of the observations with the $X M M-N e w t o n$ telescope, that is, a relatively low resolution with a high sensitivity to diffuse X-ray emission. As a result, all 


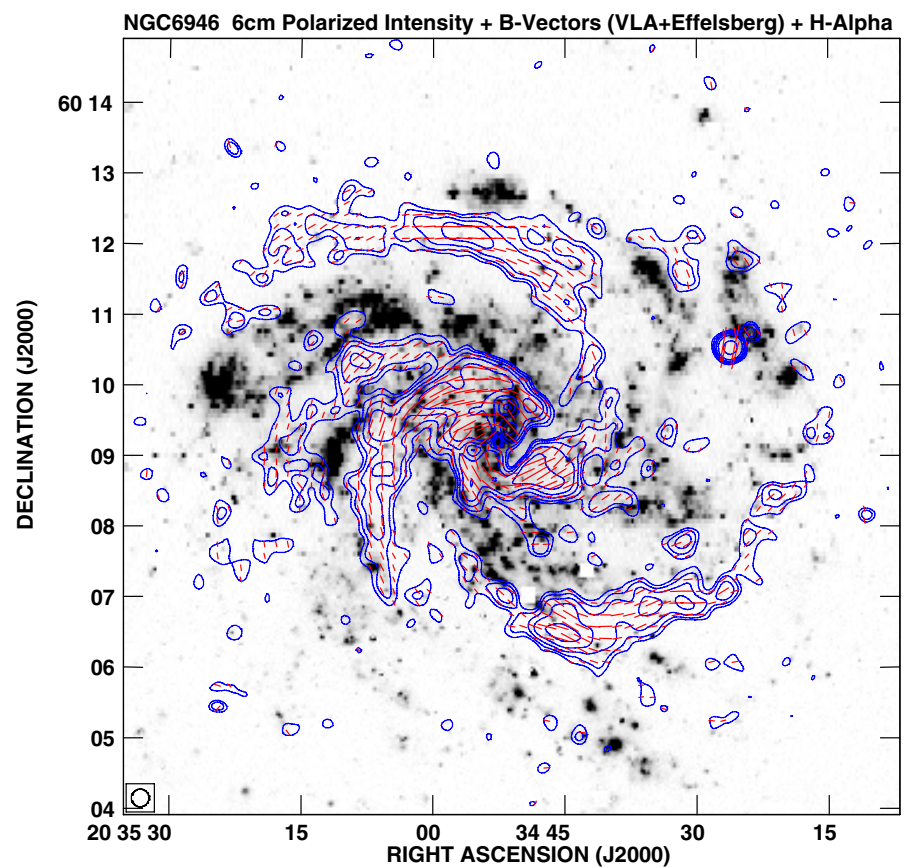

Fig. 10. Map of radio-polarized intensity at $4.85 \mathrm{GHz}(\lambda 6 \mathrm{~cm})$ of NGC 6946 (from Beck 2007). The red lines show the orientation of the magnetic fields.

Table 6. Regions in NGC 6946 used for the spectral analysis.

\begin{tabular}{cl}
\hline \hline $\begin{array}{c}\text { Region } \\
\text { letter }\end{array}$ & $\begin{array}{l}\text { Region } \\
\text { description }\end{array}$ \\
\hline A & south-western arm \\
B & north-western interarm \\
C & south-western interarm \\
D & central region w/o UV emission \\
E & south-eastern arm \\
F & eastern interarm \\
G & northeastern arm \\
H & western arms \\
I & south-eastern interarm \\
J & central region with UV emission \\
\hline
\end{tabular}

spectra of the studied point sources included significant information from the diffuse gas present across the entire disk of NGC 6946.

For several sources, an additional component to account for internal absorption was needed to obtain a good fit (see Table 4). For three sources, 19, 23, and 32, this was crucial. For source 19 , which is the core region of NGC 6946 and therefore not just a point source, this additional absorption is easy to explain because we observe the densest parts of the galaxy. The two other sources, however, are located in areas relatively free of star formation (see Fig. 4, left). Furthermore, none of the extended regions used for the spectral analysis of the diffuse emission coming from the hot gas needed an additional internal absorption component to be described by the model. Therefore, the argument of (relatively) low-resolution observations of a gas- and dust-rich galaxy cannot be used here. Instead, for each source that required an internal absorption component in the model, we
Table 7. Parameters of single plasma model-fits to the diffuse emission regions studied in NGC 6946.

\begin{tabular}{cccc}
\hline \hline Region & $\begin{array}{c}k T \\
{[\mathrm{keV}]}\end{array}$ & $\begin{array}{c}\text { Photon } \\
\text { index }\end{array}$ & $\begin{array}{c}\text { Reduced } \\
\chi^{2}\end{array}$ \\
\hline $\mathrm{A}$ & $0.53_{-0.08}^{+0.05}$ & $2.55_{-0.17}^{+0.26}$ & 1.21 \\
$\mathrm{~B}$ & $0.60_{-0.08}^{+0.06}$ & $1.84 \pm 0.22$ & 1.13 \\
$\mathrm{C}$ & $0.55_{-0.11}^{+0.10}$ & - & 1.15 \\
$\mathrm{D}$ & $0.49_{-0.14}^{+0.07}$ & $1.78_{-0.08}^{+0.13}$ & 0.99 \\
$\mathrm{E}$ & $0.53_{-0.12}^{+0.04}$ & $2.31_{-0.23}^{+0.20}$ & 1.17 \\
$\mathrm{~F}$ & $0.53 \pm 0.06$ & - & 1.05 \\
$\mathrm{G}$ & $0.50_{-0.03}^{+0.02}$ & $2.54_{-0.11}^{+0.05}$ & 1.35 \\
$\mathrm{H}$ & $0.53_{-0.03}^{+0.08}$ & $2.68_{-0.40}^{+0.45}$ & 1.24 \\
$\mathrm{I}$ & $0.50_{-0.13}^{+0.14}$ & $1.86_{-0.25}^{+0.29}$ & 1.07 \\
$\mathrm{~J}$ & $0.42_{-0.05}^{+0.09}$ & $1.69_{-0.16}^{+0.19}$ & 1.01 \\
\hline
\end{tabular}

propose that it is an X-ray binary (or an intermediate-mass black hole, IMBH) surrounded by a dust torus.

To calculate luminosities of the spectrally analysed point sources, we only used the fluxes of the power-law component to exclude the contribution from the galactic hot gas in the modelled spectrum (Table 5). Half of the sources show luminosities higher than $10^{39} \mathrm{erg} \mathrm{s}^{-1}$. This value is the most widely used observational definition of an ultra-luminous source (ULX); it is often associated with an accreting IMBH that forms in the core collapse of young dense stellar clusters (e.g. Miller \& Hamilton 2002). Since the abundance of ULXs is often linked to recent star formation activity (e.g. Berghea et al. 2013), a significant number of ULXs in NGC 6946 agrees well with the vivid star formation of this galaxy.

One of these sources is the nebula MF 16 (region 26) mentioned above. Although some of its thermal emission (from both model components) can be associated with the galactic hot gas, as mentioned above, a significant contribution from the hotter component needs a different explanation. That the gas temperature exceeds $1 \mathrm{keV}$ (Table 4), which is much higher than the temperature of the disk gas, may provide further evidence that MF16 might be indeed a supernova remnant, as previously claimed by Matonick \& Fesen (1997), and the very high temperature of the hot gas may result from shock heating of the medium surrounding the explosion region. This contradicts the findings of Berghea \& Dudik (2012), who claimed that no signs of shock heating are present.

\subsection{Extended emission}

\subsubsection{Parameters of the hot gas}

From our spectral model fits we were able to derive more parameters of the hot gas, including electron densities $n_{\mathrm{e}}$, masses $M$, thermal energies $\epsilon_{\mathrm{th}}$, and cooling times $\tau$. To perform our calculations we used the model of thermal cooling and ionization equilibrium of Nulsen et al. (1984), where $L_{\mathrm{X}}=1.11$. $\Lambda(T) n_{\mathrm{e}}^{2} V \eta, \eta$ is an unknown filling factor and $\Lambda(T)$ is a cooling coefficient of the order of $10^{-22} \mathrm{erg} \mathrm{cm}^{3} \mathrm{~s}^{-1}$ for temperatures of a few millions K (Raymond et al. 1976). However, the main difficulty in calculating the physical parameters of the hot gas component are the assumptions about the emitting volume $V$. 

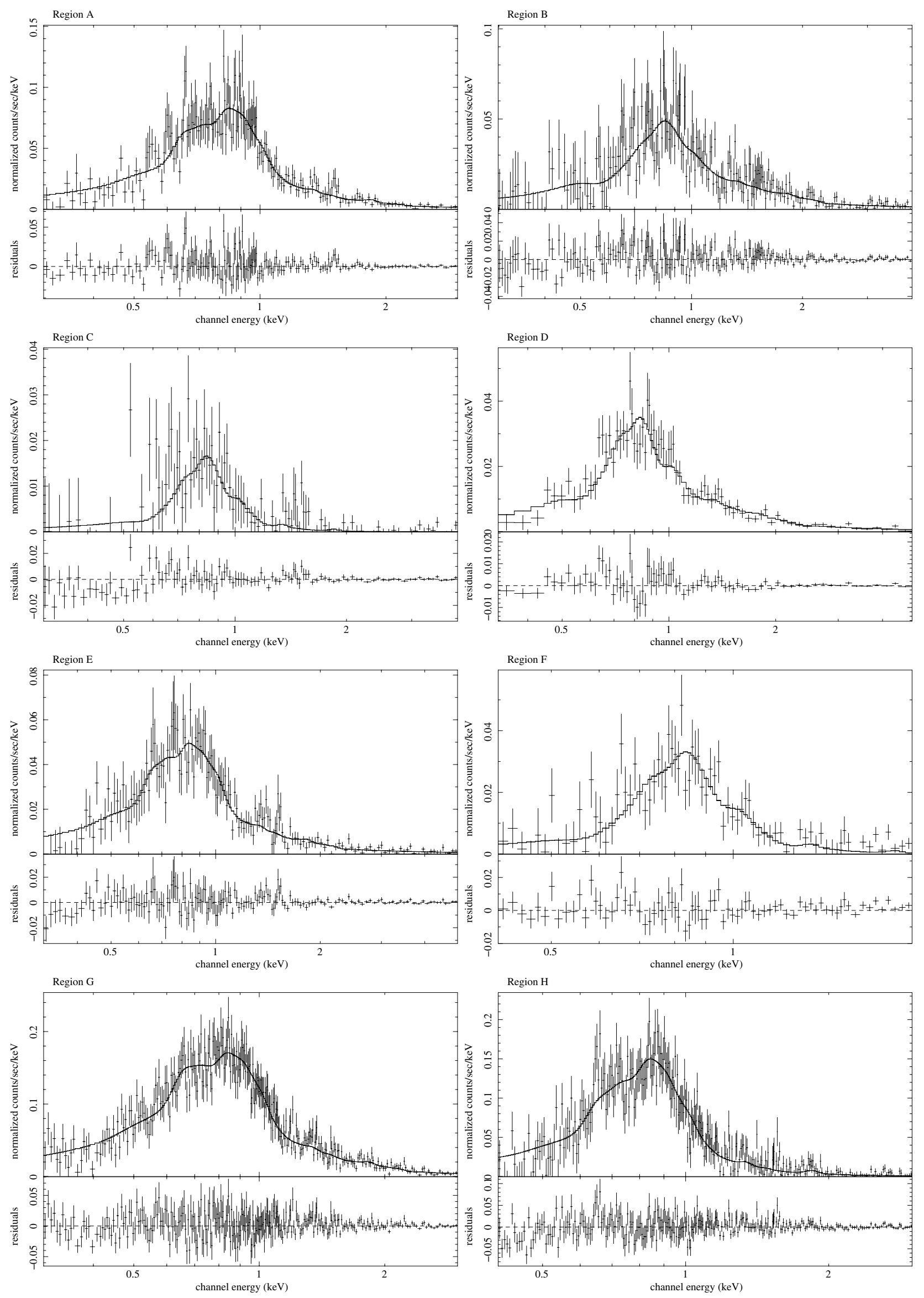

Fig. 11. Model fits to the regions of diffuse X-ray emission in NGC 6946. See Tables 8 and 9. 
Table 8. Final model-fit parameters for the regions studied in NGC 6946.

\begin{tabular}{clcccrr}
\hline \hline Region & $\begin{array}{l}\text { Model } \\
\text { type }\end{array}$ & $\begin{array}{c}k T_{1} \\
{[\mathrm{keV}]}\end{array}$ & $\begin{array}{c}k T_{2} \\
{[\mathrm{keV}]}\end{array}$ & $\begin{array}{c}\text { Photon } \\
\text { index }\end{array}$ & $\begin{array}{c}\chi_{\text {red }}^{2} \\
\begin{array}{c}\text { Net } \\
\text { counts }\end{array}\end{array}$ \\
\hline A & wabs(mekal+mekal+power law) & $0.28_{-0.04}^{+0.03}$ & $0.73_{-0.06}^{+0.07}$ & $1.82_{-0.44}^{+0.36}$ & 0.96 & 3512 \\
B & wabs(mekal+power law) & $0.60_{-0.08}^{+0.06}$ & - & $1.84 \pm 0.22$ & 1.13 & 2516 \\
C & wabs*mekal & $0.55_{-0.11}^{+0.10}$ & - & - & 1.15 & 385 \\
D & wabs(mekal+power law) & $0.49_{-0.14}^{+0.07}$ & - & $1.78_{-0.08}^{+0.13}$ & 0.99 & 1581 \\
E & wabs(mekal+mekal+power law) & $0.30_{-0.03}^{+0.05}$ & $0.80_{-0.14}^{+0.09}$ & $1.72_{-0.21}^{+0.26}$ & 0.99 & 2552 \\
F & wabs*mekal & $0.53 \pm 0.06$ & - & - & 1.05 & 896 \\
G & wabs(mekal+mekal+power law) & $0.27 \pm 0.02$ & $0.78 \pm 0.06$ & $1.93_{-0.19}^{+0.17}$ & 1.03 & 8179 \\
H & wabs(mekal+mekal+power law) & $0.28_{-0.03}^{+0.04}$ & $0.68_{-0.05}^{+0.07}$ & $1.59_{-0.88}^{+0.70}$ & 1.09 & 5008 \\
I & wabs(mekal+power law) & $0.50_{-0.13}^{+0.14}$ & - & $1.86_{-0.25}^{+0.29}$ & 1.07 & 1211 \\
J & wabs(mekal+power law) & $0.42_{-0.05}^{+0.09}$ & - & $1.69_{-0.16}^{+0.19}$ & 1.01 & 2349 \\
\hline
\end{tabular}

Notes. ${ }^{(a)}$ Column density in $\left[10^{21} \mathrm{~cm}^{-2}\right]$.
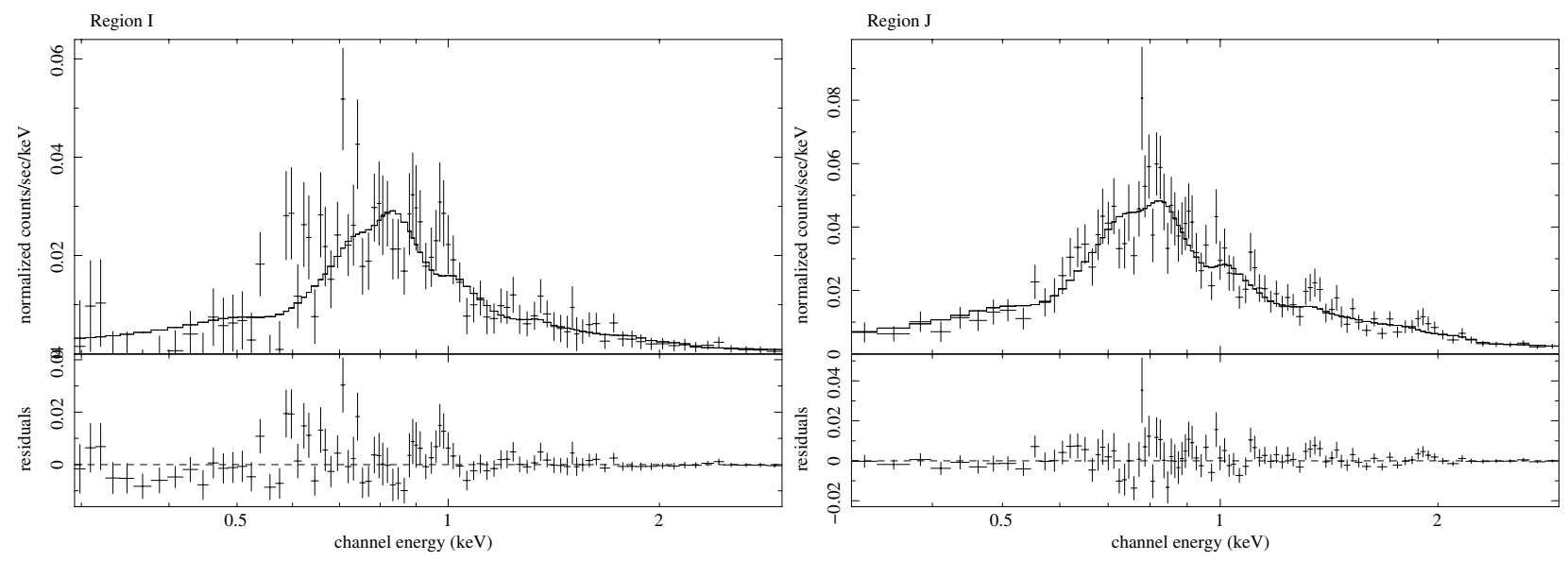

Fig. 12. Model fits to the regions of diffuse X-ray emission in NGC 6946. See Tables 8 and 9.

It seems straightforward to assume that we only see soft $\mathrm{X}$-ray emission from the visible side of the disk (and therefore the halo), with all emission from the other side being absorbed by the neutral hydrogen in the galactic disk. For the visible part of the halo emission, we assumed a cylindrical volume above the disk of NGC 6946, extending out to half of the $D_{25}$ diameter of the galaxy $(10 \mathrm{kpc})$. In this geometry, the halo around NGC 6946 would be roughly spherical. This approach seems to be justified for a starbust galaxy that has significantly extended halos of the $\mathrm{X}$-ray emission. For the disk emission we assumed a disk thickness of $1 \mathrm{kpc}$. For the interarm regions, for which a single thermal model was fitted, we used volumes of $10 \mathrm{kpc}$ times the area of a region, as the model describes the mixed emission from both the disk and the halo (see Sect. 3.2.2).

Tables 10 and 11 present the derived parameters of the hot gas in the areas of diffuse emission in NGC 6946.

To verify the obtained values, a comparison with earlier Chandra observations of NGC 6946 by Schlegel et al. (2003) would be an important step. Because of the low sensitivity to diffuse emission of these observations, the analysis was unfortunately made only for the entire galactic disk and no detailed study is available. Still, their temperatures of $0.25 \pm 0.03 \mathrm{keV}$ and $0.70 \pm 0.10 \mathrm{keV}$ for two thermal components agree very well with our values for the halo and the disk gas temperatures, respectively. The electron density of $\sim 0.012\left(\times \eta^{-0.5}\right) \mathrm{cm}^{-3} \mathrm{de}-$ rived by Schlegel et al. (2003) for the $1 \mathrm{kpc}$ thick disk also matches our results well if we consider a ten times larger volume than used for our calculations. A similar study of six other nearby face-on late-type spiral galaxies was reported by Owen \& Warwick (2009), who obtained temperatures of two thermal model fits of $0.2-0.3 \mathrm{keV}$ and $0.6-0.7 \mathrm{keV}$ and the derived electron densities of the gas of the order of a few $10^{-3}\left(\times \eta^{-0.5}\right) \mathrm{cm}^{-3}$, depending on the level of the star-forming activity.

\subsubsection{Hot gas components}

As mentioned before, for the regions of the spiral arms we needed a two-temperature model to account for the emission from the hot gas residing in both galactic disk and the surrounding halo. Although the temperatures of the hot gas are almost identical for the halo components and similar for the disk components (where two pairs could be distinguished - A with $\mathrm{H}$ and E with $\mathrm{G}$ - see Table 8), the derived parameters show significant differences (see Table 11). As expected, for the north-eastern spiral arm with the brightest $\mathrm{H} \alpha$ and UV emission, marked as region $\mathrm{G}$, we obtained the highest values of number density and energy density of all spiral arms. 
Table 9. Total (0.3-12 keV) unabsorbed fluxes in $10^{-14} \mathrm{erg} \mathrm{cm}^{-2} \mathrm{~s}^{-1}$ for modelled regions in NGC 6946.

\begin{tabular}{ccccc}
\hline \hline Region & Mekal cold & Mekal hot & Power law & Total \\
\hline A & $3.5_{-0.9}^{+0.8}(0.32)$ & $2.5_{-0.6}^{+0.8}(0.23)$ & $4.8_{-2.0}^{+5.1}(0.45)$ & $10.8_{-3.5}^{+6.7}$ \\
B & $2.4_{-0.5}^{+0.3}(0.22)$ & - & $8.5_{-2.5}^{+4.0}(0.78)$ & $10.9_{-2.9}^{+4.4}$ \\
C & $1.1_{-0.2}^{+0.1}(1.00)$ & - & - & $1.1_{-0.2}^{+0.1}$ \\
D & $1.4_{-0.3}^{+0.2}(0.25)$ & - & $4.2_{-1.1}^{+1.0}(0.75)$ & $5.6_{-1.4}^{+1.2}$ \\
E & $2.6^{+0.9}(0.26)$ & $1.6_{-0.6}^{+0.7}(0.16)$ & $5.7_{-2.2}^{+3.5}(0.58)$ & $9.8_{-3.74}^{+5.0}$ \\
F & $2.1_{-0.2}^{+0.1}(1.00)$ & - & - & $2.1_{-0.2}^{+0.1}$ \\
G & $9.3_{-1.4}^{+1.5}(0.31)$ & $5.2_{-1.3}^{+1.0}(0.18)$ & $15.3_{-3.3}^{+5.4}(0.51)$ & $29.7_{-6.0}^{+7.8}$ \\
H & $6.6_{-1.7}^{+1.9}(0.43)$ & $6.4_{-1.8}^{+1.5}(0.42)$ & $2.3_{-1.9}^{+12.8}(0.15)$ & $15.4_{-5.5}^{+16.0}$ \\
I & $1.2 \pm 0.2(0.30)$ & - & $2.8_{-1.1}^{+1.7}(0.70)$ & $4.0_{-1.3}^{+1.8}$ \\
J & $2.0 \pm 0.5(0.21)$ & - & $7.5_{-1.8}^{+2.6}(0.79)$ & $9.5_{-2.3}^{+3.1}$ \\
\hline
\end{tabular}

Notes. Values in parentheses are fractions of the total flux for a given component.

Table 10. Derived parameters of the hot gas in the interarm and central regions of NGC 6946.

\begin{tabular}{cccccc}
\hline \hline Region & $\begin{array}{c}\left(n_{\text {halo }} \eta^{-0.5}\right)^{a} \\
{\left[10^{-3} \mathrm{~cm}^{-3}\right]}\end{array}$ & $\begin{array}{c}\left(M_{\mathrm{gas}}^{\text {halo }} \eta^{0.5}\right)^{a} \\
{\left[10^{6} M_{\odot}\right]}\end{array}$ & $\begin{array}{c}\left(E_{\text {th }}^{\text {halo }} \eta^{0.5}\right)^{a} \\
{\left[10^{54} \mathrm{erg}\right]}\end{array}$ & $\begin{array}{c}\left(\epsilon_{\mathrm{th}}^{\text {halo }} \eta^{-0.5}\right)^{a} \\
{\left[10^{-12} \mathrm{erg} \mathrm{cm}^{-3}\right]}\end{array}$ & $\begin{array}{c}\left(\tau^{\text {halo }} \eta^{0.5}\right)^{a} \\
{[\mathrm{Myr}]}\end{array}$ \\
\hline B & $0.84 \pm 0.07$ & $5.09_{-0.47}^{+0.37}$ & $8.74_{-1.87}^{+1.58}$ & $1.22_{-0.26}^{+0.22}$ & $1969_{-13}^{+98}$ \\
C & $0.72_{-0.06}^{+0.07}$ & $2.66_{-0.24}^{+0.26}$ & $4.20_{-1.14}^{+1.24}$ & $0.96_{-0.26}^{+0.28}$ & $2063_{-277}^{+388}$ \\
D & $2.03_{-0.12}^{+0.15}$ & $1.22_{-0.07}^{+0.09}$ & $1.71_{-0.56}^{+0.39}$ & $2.40_{-0.79}^{+0.54}$ & $662_{-96}^{+48}$ \\
F & $1.22 \pm 0.06$ & $3.08_{-0.16}^{+0.15}$ & $4.67_{-0.74}^{+0.79}$ & $1.56_{-0.25}^{+0.26}$ & $1202_{-84}^{+139}$ \\
I & $1.22_{-0.12}^{+0.11}$ & $1.86_{-0.18}^{+0.16}$ & $2.66_{-0.88}^{+1.05}$ & $1.47_{-0.49}^{+0.58}$ & $1107_{-146}^{+325}$ \\
J & $2.63_{-0.28}^{+0.22}$ & $1.48_{-0.16}^{+0.12}$ & $1.78_{-0.38}^{+0.56}$ & $2.66_{-0.57}^{+0.84}$ & $480_{-24}^{+25}$ \\
\hline
\end{tabular}

Notes. ${ }^{(a)} \eta$ is the volume filling factor. The columns are the region name, electron number density, total gas mass, total thermal energy, thermal energy density, and cooling time.

\subsubsection{Magnetic fields in NGC 6946}

To analyse the magnetic field parameters we used the same regions as for the spectral analysis. For each region both total intensity and polarized intensity fluxes at a radio wavelength of $6.2 \mathrm{~cm}$ with a beam of $15^{\prime \prime}$ were obtained. Then, using the energy equipartition formula provided by Beck \& Krause (2005), we calculated the strengths of both the total and ordered magnetic fields. The calculations were made assuming a synchrotron spectral index of 1.0, an inclination of the galactic disk of $30^{\circ}$, and a proton-to-electron ratio of 100 . For the emitting volume a disk of $1 \mathrm{kpc}$ thickness was assumed. The main uncertainties are introduced by the last two parameters. They may vary by a factor of 2. With the assumed spectral index this amounts to an error of $\sim 30 \%$ for the strength of the magnetic field and $\sim 60 \%$ for its energy density. We note here, however, that such errors are systematic, which means that we may in fact expect the relative uncertainties between the points to be smaller. Table 12 summarizes our results and also provides values for the energy densities of the magnetic field.

Apart from the central regions of the galaxy (regions D and J), the strength of the total magnetic field is roughly constant across the disk. A slight increase can be observed in the most prominent spiral arms (regions A and G). Consequently, these regions show higher energy densities of the magnetic field, with the maximum near the galactic core. The strengths of the ordered magnetic field are also similar in all parts of the disk, with higher values in the areas of the magnetic arms and the central region of the galaxy. As suggested by Fig. 10, the areas of the magnetic arms show a much higher degree of polarization than the other regions.

\subsubsection{Hot gas and magnetic fields of spiral and magnetic arms}

In addition to the grand-design structure of its gaseous spiral arms, NGC 6946 presents a distinct spiral structure of the magnetic fields which resembles magnetic arms that are phaseshifted with regard to the gaseous ones. These magnetic arms coincide well with the interarm regions. Since the spiral arm and interarm regions vary significantly in terms of the ISM structure, we investigated the emission from the hot gas in both areas to obtain more clues about the interplay of the magnetic field and the hot plasma. Because a model with two thermal components was used to analyse the emission from the spiral arms (accounting for the disk and halo components), to compare it with the emission from the hot gas in and above the interarm (magnetic arm) areas, we needed to calculate averages of the values obtained from the two-temperature fits. 
Table 11. Derived parameters of the hot gas in and above the spiral arm regions of NGC 6946.

\begin{tabular}{cccccc}
\hline \hline Region & $\begin{array}{c}\left(n_{\text {disk }} \eta^{-0.5}\right)^{a} \\
{\left[10^{-3} \mathrm{~cm}^{-3}\right]}\end{array}$ & $\begin{array}{c}\left(M_{\text {gas }}^{\text {disk }} \eta^{0.5}\right)^{a} \\
{\left[10^{6} M_{\odot}\right]}\end{array}$ & $\begin{array}{c}\left(E_{\text {th }}^{\text {disk }} \eta^{0.5}\right)^{a} \\
{\left[10^{54} \mathrm{erg}\right]}\end{array}$ & $\begin{array}{c}\left(\epsilon_{\text {th }}^{\text {dis }} \eta^{-0.5}\right)^{a} \\
{\left[10^{-12} \mathrm{erg} \mathrm{cm}^{-3}\right]}\end{array}$ & $\begin{array}{c}\left(\tau^{\text {halo }} \eta^{0.5}\right)^{a} \\
{[\mathrm{Myr}]}\end{array}$ \\
\hline$A_{\text {disk }}$ & $4.13_{-0.40}^{+0.52}$ & $1.14_{-0.11}^{+0.15}$ & $2.39_{-0.41}^{+0.56}$ & $7.25_{-1.24}^{+1.69}$ & $517_{-34}^{+47}$ \\
$A_{\text {halo }}$ & $1.69_{-0.19}^{+0.15}$ & $4.68_{-0.54}^{+0.42}$ & $3.75_{-0.91}^{+0.78}$ & $1.14_{-0.28}^{+0.24}$ & $579_{-10}^{+12}$ \\
$E_{\text {disk }}$ & $2.92_{-0.49}^{+0.47}$ & $1.05_{-0.18}^{+0.17}$ & $2.41_{-0.76}^{+0.70}$ & $5.62_{-1.77}^{+1.63}$ & $815_{-83}^{+79}$ \\
$E_{\text {halo }}$ & $1.26_{-0.24}^{+0.16}$ & $4.53_{-0.87}^{+0.56}$ & $3.89_{-1.06}^{+1.21}$ & $0.91_{-0.25}^{+0.28}$ & $809_{-22}^{+90}$ \\
$G_{\text {disk }}$ & $4.63_{-0.55}^{+0.31}$ & $2.15_{-0.26}^{+0.14}$ & $4.79_{-0.90}^{+0.72}$ & $8.70_{-1.62}^{+1.30}$ & $498_{-18}^{+42}$ \\
$G_{\text {halo }}$ & $2.15 \pm 0.01$ & $9.96 \pm 0.07$ & $7.70_{-0.62}^{+0.63}$ & $1.40 \pm 0.11$ & $448_{-31}^{+37}$ \\
$H_{\text {disk }}$ & $3.65_{-0.49}^{+0.37}$ & $3.19_{-0.42}^{+0.32}$ & $6.21_{-1.22}^{+1.33}$ & $5.95_{-1.17}^{+1.28}$ & $524_{-8}^{+63}$ \\
$H_{\text {halo }}$ & $1.31_{-0.16}^{+0.14}$ & $11.44_{-1.43}^{+1.22}$ & $9.18_{-2.01}^{+1.34}$ & $0.88_{-0.19}^{+0.13}$ & $752_{-83}^{+39}$ \\
\hline
\end{tabular}

Notes. ${ }^{(a)} \eta$ is the volume filling factor. The columns are the region name, electron number density, total gas mass, total thermal energy, thermal energy density, and cooling time.

Table 12. Properties of the magnetic fields in NGC 6946.

\begin{tabular}{cccccc}
\hline \hline Region & $\begin{array}{c}S_{\text {synch }} \\
{[\mathrm{mJy} / \mathrm{beam}]}\end{array}$ & $\begin{array}{c}p_{\text {synch }} \\
{[\%]}\end{array}$ & $\begin{array}{c}B_{\text {tot }} \\
{[\mu \mathrm{G}]}\end{array}$ & $\begin{array}{c}\epsilon_{\mathrm{B}} \\
{\left[10^{-12} \mathrm{erg} \mathrm{cm}^{-3}\right]}\end{array}$ & $\begin{array}{c}B_{\text {ord }} \\
{[\mu \mathrm{G}]}\end{array}$ \\
\hline $\mathrm{A}$ & 0.67 & 7.7 & $17.2 \pm 5.2$ & $11.8 \pm 7.1$ & $4.9 \pm 1.5$ \\
$\mathrm{~B}$ & 0.36 & 22.6 & $14.4 \pm 4.3$ & $8.3 \pm 5.0$ & $7.2 \pm 2.2$ \\
$\mathrm{C}$ & 0.31 & 26.3 & $13.8 \pm 4.1$ & $7.6 \pm 4.6$ & $7.4 \pm 2.2$ \\
$\mathrm{D}$ & 1.74 & 9.3 & $21.8 \pm 6.5$ & $18.9 \pm 11.3$ & $6.8 \pm 2.0$ \\
$\mathrm{E}$ & 0.42 & 12.7 & $15.2 \pm 4.6$ & $9.1 \pm 5.5$ & $5.6 \pm 1.7$ \\
$\mathrm{~F}$ & 0.39 & 16.5 & $14.8 \pm 4.4$ & $8.8 \pm 5.3$ & $6.2 \pm 1.9$ \\
$\mathrm{G}$ & 0.54 & 6.5 & $16.3 \pm 4.9$ & $10.6 \pm 6.4$ & $4.2 \pm 1.3$ \\
$\mathrm{H}$ & 0.39 & 10.8 & $14.9 \pm 4.5$ & $8.9 \pm 5.3$ & $5.0 \pm 1.5$ \\
$\mathrm{I}$ & 0.35 & 20.5 & $14.3 \pm 4.3$ & $8.2 \pm 4.9$ & $6.8 \pm 2.0$ \\
$\mathrm{~J}$ & 1.40 & 14.6 & $20.4 \pm 6.1$ & $16.6 \pm 10.0$ & $8.1 \pm 2.4$ \\
\hline
\end{tabular}

Notes. The columns are the region name, non-thermal radio flux, degree of polarization, total magnetic field strength, magnetic field energy, and ordered magnetic field strength.

We added gas masses and thermal energies for the appropriate regions. Next, we calculated number and energy densities, taking into account the volumes assumed for the disk and halo component emission (the area of a given region times 1 or $10 \mathrm{kpc}$, respectively). Our results are presented in Table 13. We compared the averaged values of number and energy densities for all spiral arm regions with respective values for the interarm regions. We also calculated the ratios of the thermal energy densities to number densities, hence obtaining an average energy per particle, which is independent of the unknown volume-filling factor $\left(E_{\mathrm{p}}=\epsilon / n\right)$.

We present our results in Table 14. The interarm regions show higher values of an energy per particle than for the spiral arm regions. This is consistent with the single-temperature fits (Fig. 7), which show slightly higher temperatures for the regions of the magnetic arms. This means that there may be an additional effect that provides thermal energy to the interarm regions. Since this additional heating might also be due to magnetic reconnection, we analysed the magnetic field properties of the areas of the spiral arms and the interarm regions.

\subsubsection{Heating of the gas by magnetic reconnection?}

Because for the interarm regions we have the information about the hot gas coming from both the disk and the above halo (onetemperature fit to the spectra), a direct comparison of the energy densities of disk hot gas and magnetic fields is possible for the regions of the spiral arms alone. Nevertheless, since we are interested in the global energy budget of the galaxy, we need information about both thermal and magnetic energy densities for the disk and the halo. Although halo magnetic fields surely exist (as observed in edge-on spiral galaxies), we do not have any direct information on their structure and strength in the case of NGC 6946. The observed radio emission is, however, an integration along the line of sight, that is, we see contributions from both the galaxy disk and halo. Since the majority of the cosmic rays originates in the underlying disk, an assumption for the emitting volume (the disk) seems to be justified. As the vertical scale height of the halo magnetic fields of $\sim 6-7 \mathrm{kpc}$ (e.g. Beck 2015) is similar to the assumed size of the hot gas halo $(10 \mathrm{kpc})$, we do not expect a significant change of the magnetic energy density in the halo, especially when an uncertainty of its 
Table 13. Averaged parameters of the hot gas in and above the spiral arm regions of NGC 6946.

\begin{tabular}{ccccc}
\hline \hline Region & $\begin{array}{c}\left(n \eta^{-0.5}\right)^{a} \\
{\left[10^{-3} \mathrm{~cm}^{-3}\right]}\end{array}$ & $\begin{array}{c}\left(M_{\mathrm{gas}} \eta^{0.5}\right)^{a} \\
{\left[10^{6} M_{\odot}\right]}\end{array}$ & $\begin{array}{c}\left(E_{\mathrm{th}} \eta^{0.5}\right)^{a} \\
{\left[10^{54} \mathrm{erg}\right]}\end{array}$ & $\begin{array}{c}\left(\epsilon_{\mathrm{th}} \eta^{-0.5}\right)^{a} \\
{\left[10^{-12} \mathrm{erg} \mathrm{cm}^{-3}\right]}\end{array}$ \\
\hline $\mathrm{A}$ & $1.91_{-0.21}^{+0.19}$ & $5.82_{-0.66}^{+0.57}$ & $6.14_{-1.32}^{+1.34}$ & $1.70_{-0.38}^{+0.37}$ \\
$\mathrm{E}$ & $1.41_{-0.27}^{+0.18}$ & $5.58_{-1.05}^{+0.72}$ & $6.30_{-1.82}^{+1.91}$ & $1.34_{-0.39}^{+0.40}$ \\
$\mathrm{G}$ & $2.38_{-0.06}^{+0.03}$ & $12.11_{-0.33}^{+0.21}$ & $12.49_{-1.51}^{+1.35}$ & $2.06_{-0.25}^{+0.22}$ \\
$\mathrm{H}$ & $1.52_{-0.19}^{+0.16}$ & $14.63_{-1.85}^{+1.54}$ & $15.39_{-3.22}^{+2.65}$ & $1.35_{-0.29}^{+0.23}$ \\
\hline
\end{tabular}

Notes. ${ }^{(a)} \eta$ is the volume filling factor. The columns are the region name, electron number density, total gas mass, total thermal energy, and thermal energy density.

Table 14. Thermal energy per particle and energy densities of the magnetic field for regions of the spiral and magnetic arms of NGC 6946.

\begin{tabular}{ccrccr}
\hline \hline Spiral arm & $E_{\mathrm{p}}$ & $\epsilon_{\mathrm{B}}$ & Magnetic arm & $E_{\mathrm{p}}$ & $\epsilon_{\mathrm{B}}$ \\
\hline $\mathrm{A}$ & $0.89_{-0.11}^{+0.10}$ & $11.8 \pm 7.1$ & $\mathrm{~B}$ & $1.45_{-0.15}^{+0.14}$ & $8.3 \pm 5.0$ \\
$\mathrm{E}$ & $0.95_{-0.12}^{+0.14}$ & $9.1 \pm 5.5$ & $\mathrm{C}$ & $1.33_{-0.27}^{+0.23}$ & $7.6 \pm 4.6$ \\
$\mathrm{G}$ & $0.87_{-0.09}^{+0.08}$ & $10.6 \pm 6.4$ & $\mathrm{~F}$ & $1.28_{-0.16}^{+0.15}$ & $8.8 \pm 5.3$ \\
$\mathrm{H}$ & $0.88_{-0.08}^{+0.06}$ & $8.9 \pm 5.3$ & $\mathrm{I}$ & $1.20_{-0.31}^{+0.34}$ & $8.2 \pm 4.9$ \\
\hline
\end{tabular}

Notes. $E_{\mathrm{p}}$ - energy per particle in $10^{-9} \mathrm{erg} ; \epsilon_{B}$ - magnetic energy density in $10^{-12} \mathrm{erg} \mathrm{cm}^{-3}$.

calculation $(60 \%)$ is considered. It is therefore justified to compare the obtained magnetic energy densities with those of the hot gas in and above the interarm regions.

Still, for all areas of the disk of NGC 6946 we see much higher energy densities of the magnetic fields than those of the disk component of the hot gas (Tables 11 and 12). Interestingly, for the region of the most prominent spiral arm (region G), conditions closest to equilibrium are observed, with the energy density of the magnetic field only $22 \%$ higher than that of the hot gas in the galactic disk. For the remaining spiral arms this difference is as high as $50-63 \%$. If we compare the magnetic field energy densities to those of the halo (Tables 10 and 11) or averaged values for the hot gas in and above the spiral arms (disk+halo, Table 13), a distinct dominance of magnetic fields by a factor of a few is visible. This suggests that only in the areas of high starforming activity it is possible that the thermal energy density of the gas is similar to that of the local magnetic fields.

To investigate the interplay between the thermal gas and the magnetic fields in greater detail, we compared the averaged (i.e. from both disk and halo components) thermal energies per particle with the magnetic field energy densities for the spiral and magnetic arms. We calculated the average values for the gaseous spiral arms and the magnetic arms, which resulted in an energy density of the magnetic field of about $10.1 \times 10^{-12} \mathrm{erg} \mathrm{cm}^{-3}$ for the spiral arms and $8.2 \times 10^{-12} \mathrm{erg} \mathrm{cm}^{-3}$ for the interarm regions and for energies per particle of $0.90 \times 10^{-9} \mathrm{erg}$ and $1.32 \times$ $10^{-9} \mathrm{erg}$, respectively. Our results are presented in Table 14. An anti-correlation between the magnetic field strength (and its energy) and the thermal energy of the gas is visible (Fig. 13); with a slight decrease of the energy density of the magnetic field (by 23\%), the energy per particle increases significantly (by 68\%).

A possible explanation is that in regions that simultaneously show higher thermal energies of the gas and lower energies of the magnetic fields, some of the energy of the magnetic field might have been converted into thermal energy by

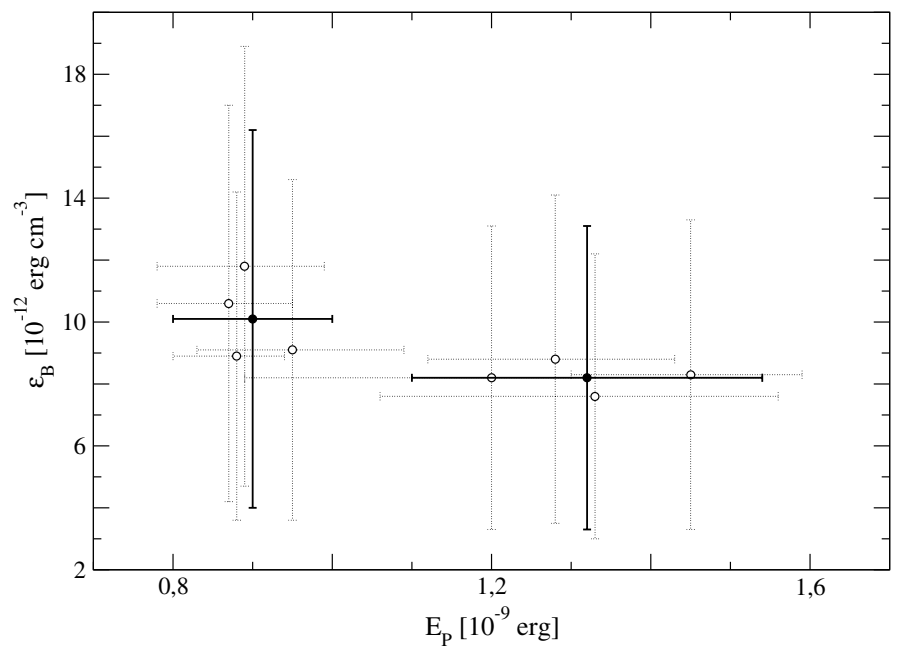

Fig. 13. Relation between energy per particle $\left(E_{\mathrm{P}}\right)$ and energy density of the magnetic field $\left(\epsilon_{\mathrm{B}}\right)$ in the spiral and the magnetic arms of NGC 6946. Open symbols with dashed error bars present each spiral arm (left side of the plot) and magnetic arm (right side of the plot). Filled symbols with solid error bars present average values of the respective data points.

magnetic reconnection. Fast reconnection should be possible in most astrophysical plasmas (Hanasz \& Lesch 2003), with a heating rate proportional to the Alfvén speed (Lesch \& Bender 1990; Lazarian et al. 2015). As the gas density in the interarm regions is lower while the total magnetic field is almost as strong as in the spiral arms, the Alfvén speed is higher, and hence the heating rate is higher in interarm regions. Indeed, we do observe such an additional heating of the gas in the magnetic arm regions (see Tables 7 and 8).

In general, such a slight increase in temperature in the interarm regions could be easily explained by longer cooling times that are due to the lower density of the gas. However, if we 


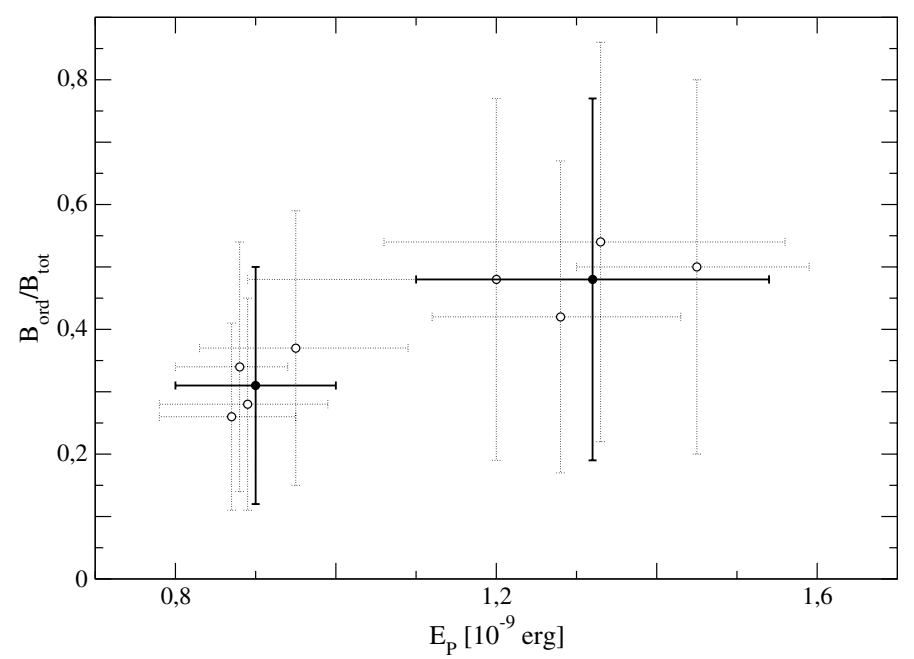

Fig. 14. Relation between energy per particle $\left(E_{\mathrm{P}}\right)$ and regularity $\left(B_{\text {ord }} / B_{\text {tot }}\right)$ of the magnetic field in the spiral and the magnetic arms of NGC 6946. Symbols are the same as used in Fig. 13.

compare interarm regions $\mathrm{B}$ and $\mathrm{C}$ with $\mathrm{F}$ and $\mathrm{I}$, we note that in magnetic arms $\mathrm{B}$ and $\mathrm{C}$ higher temperatures, lower number and energy densities than in magnetic arms $\mathrm{F}$ and I were obtained. Surprisingly, the difference in cooling times reaches almost a factor of 2. Still, for all four interarm regions practically the same magnetic field strengths and energy densities were observed. The difference is visible, however, when ordered fields are considered - in both regions $\mathrm{B}$ and $\mathrm{C}$ we see a much higher regularity of the field (i.e. the ratio of the strengths of ordered and total magnetic field). This trend is visible in all magnetic arms when compared to the spiral arms - the regularity of the magnetic field increases with the energy per particle (Fig. 14).

The above findings allow constructing a picture of both turbulence and reconnection acting in a galactic disk. Although in the spiral arms reconnection effects are expected to be more efficient (stronger field tangling), their action might be difficult to see because both the heating and field (dis)ordering is dominated by turbulence. In the interarm regions, however, where the magnetic field is highly ordered, reconnection heating may dominate turbulence heating, and the increase in temperature due to reconnection heating might be noticeable. This is what we observe, especially in the magnetic arms $\mathrm{B}$ and $\mathrm{C}$, which have slightly higher temperatures than most star-forming spiral arms. Although reconnection is a very local process, acting at distances of a few pc or less, if it is equally efficient throughout the entire magnetic arm, it might contribute to the field ordering, an effect that would last longer because of the weaker turbulence. Again, this high field ordering is most distinctly seen in magnetic arms B and C.

The region of the western spiral arm (region $\mathrm{H}$ ) is interesting. While for the other spiral arm regions we observe a significant contribution from the halo component (see Table 9), region $\mathrm{H}$ shows almost equal contributions from the halo and disk components. Furthermore, the difference of temperatures of the two components is the lowest in this region, owing mainly to the lowest temperature of all spiral arm disk components. This may suggest that for the western spiral arm mixing of the disk and the halo gas is the most efficient. Since the southern part of region $\mathrm{H}$ is in the area of strong Faraday depolarization, as reported by Beck (1991, 2007), this might be a piece of evidence for vertical magnetic fields and enhanced outflow speed in this area of the galactic disk. In particular the region of the south-western spiral $\operatorname{arm}($ region A), which also contributes to the depolarization area, shows a similar trend for the interplay of the halo and disk gas components, although at a lower level.

\section{Summary and conclusions}

The detailed analysis of the X-ray emission from the hot gas in NGC 6946, together with earlier radio continuum studies, can be summarized as follows:

- A spectral analysis of the point sources revealed a significant number of ULXs, which agrees with the enhanced star formation of the galaxy.

- The galaxy presents significant emission from the hot gas across its entire star-forming disk. Intensity enhancements are found in the regions of the spiral arms, also in the harder energy band. A significant amount of the very soft emission is found in the region of high Faraday depolarization.

- The radio-polarized emission structure is reflected in the spectral properties of the hot gas - areas of magnetic arms visible in the interarm regions are well described with a single thermal plasma model, which shows that the temperature of the hot gas is slightly higher than in the spiral arm regions.

- An increase in temperature of the hot gas in the magnetic arm regions could be described as additional heating due to magnetic reconnection.

- A possible conversion of magnetic field energy into thermal energy of the hot gas in the interarm regions is suggested by the lower energy density and strength of the magnetic field and the higher thermal energy per particle, when compared to the areas of the spiral arms.

- In the conditions of low turbulence in the magnetic arm regions, reconnection, acting mostly on tangled fields, might also contribute to the field ordering, as suggested by both the highest temperatures of the hot gas and the highest degree of polarization in magnetic arms B and $\mathrm{C}$.

- We found signatures of a very hot gas in the area of the ultraluminous source MF 16, which may suggest shock heating of the gas by a supernova explosion.

Acknowledgements. We thank Wolfgang Pietsch and Stefania Carpano for their collaboration on the original XMM-Newton observing proposals that form the base of this paper. Special thanks go to Harald Lesch and Alex Lazarian, who improved our understanding of the reconnection theory. We also thank Stefanie Komossa for useful comments on an earlier version of the paper, and the anonymous referee for a helpful report.

\section{References}

Arnaud, K. A. 1996, in Astronomical Data Analysis Software and Systems V, eds. G. Jacoby, \& J. Barnes, ASP Conf. Ser., 101, 17

Beck, R. 1991, A\&A, 251, 15

Beck, R. 2004, Ap\&SS, 289, 293

Beck, R. 2007, A\&A, 470, 539

Beck, R. 2015, A\&A, 578, A93

Beck, R., \& Hoernes, P. 1996, Nature, 379, 47

Beck, R., \& Krause, M. 2005, Astron. Nachr., 326, 414

Berghea, C. T., \& Dudik, R. P. 2012, ApJ, 751, 104

Berghea, C. T., Dudik, R. P., Tincher, J., \& Winter, L. M. 2013, ApJ, 776, 100

Carter, J. A., \& Read, A. M. 2007, A\&A, 464, 1155

Chamandy, L., Subramanian, K., \& Shukurov, A. 2013a, MNRAS, 428, 3569

Chamandy, L., Subramanian, K., \& Shukurov, A. 2013b, MNRAS, 433, 3274

Chamandy, L., Shukurov, A., \& Subramanian, K. 2015, MNRAS, 446, L6

Ehle, M., \& Beck, R. 1993, A\&A, 273, 45

Frick, P., Beck, R., Shukurov, A., et al. 2000, MNRAS, 318, 925

Fridriksson, J. K., Homan, J., Lewin, W. H. G., Kong, A. K. H., \& Pooley, D. 2008, ApJS, 177, 465 
A\&A 585, A3 (2016)

Gabriel, C., Denby, M., Fyfe, D. J., et al. 2004, ASP Conf. Ser., 314, 759 Hanasz, M., \& Lesch, H. 2003, A\&A, 404, 389

Holt, S. S., Schlegel, E. M., Hwang, U., \& Petre, R. 2003, ApJ, 588, 792 Israel, P. F. 1980, A\&A, 90, 246

Jansen, F., Lumb, D., Altieri, B., et al. 2001, A\&A, 365, 1

Kaastra, J. S. 1992, An X-Ray Spectral Code for Optically Thin Plasmas (Internal SRON-Leiden Report, updated version 2.0)

Kajava, J. J. E., \& Poutanen, J. 2009, MNRAS, 398, 1450

Kalberla, P. M. W., Burton, W. B., Hartmann, D., et al. 2005, A\&A, 440, 775

Lazarian, A., Eyink, G., Vishniac, E., \& Kowal, G. 2015, Phil. Trans. Roy. Soc. London Ser. A, 373, 40144

Lesch, H., \& Bender, R. 1990, A\&A, 233, 417

Liu, J. 2011, ApJS, 192, 55

Matonick, D. M., \& Fesen, R. A. 1997, ApJS, 112, 49

Mewe, R., Gronenschild, E. H. B. M., \& van den Oord, G. H. J. 1985, A\&AS, 62, 197

Miller, M. C., \& Hamilton, D. P. 2002, MNRAS, 330, 232

Molendi, S., De Luca, A., \& Leccardi, A. 2004, A\&A, 419, 837

Moss, D., Beck, R., Sokoloff, D., et al. 2013, A\&A, 556, A147
Moss, D., Stepanov, R., Krause, M., Beck, R., \& Sokoloff, D. 2015, A\&A, 578, A94

Nieten, C., Dumke, M., Beck, R., \& Wielebinski, R. 1999, A\&A, 347, L5

Nulsen, P. E. J., Stewart, G. C., \& Fabian, A. C. 1984, MNRAS, 208, 185

Owen, R. A., \& Warwick, R. S. 2009, MNRAS, 394, 1741

Paturel, G. 2003, A\&A, 412, 45

Pietsch, W., Misanovic, Z., Haberl, F., et al. 2004, A\&A, 426, 11

Raymond, J. C., Cox, D. P., \& Smith, B. W. 1976, ApJ, 204, 290

Schlegel, E. M. 1994a, ApJ, 424, L99

Schlegel, E. M. 1994b, ApJ, 434, 523

Schlegel, E. M. 1994c, AJ, 108, 1893

Schlegel, E. M., Holt, S. S., \& Petre, R. 2003, ApJ, 598, 982

Soria, R., \& Perna, R. 2008, ApJ, 683, 767

Strüder, L., Briel, U., Dennerl., K., et al. 2001, A\&A, 365, 18

Telesco, C. M., \& Harper, D. A. 1980, ApJ, 235, 392

Tüllmann, R., Pietsch, W., Rossa, J., Breitschwerdt, D., \& Dettmar, R.-J. 2006, A\&A, 448, 43

Turner, M. J. L., Abbey, A., Arnaud, M., et al. 2001, A\&A, 365, 27

Walsh, W., Beck, R., Thuma, G., et al. 2002, A\&A, 388, 7 